\title{
An Ultra-Broadband Dielectric and Optical Kerr-Effect Study of the Ionic Liquids Ethyl- and Propylammonium Nitrate
}

Thomas Sonnleitner, ${ }^{\dagger}$ David A. Turton, ${ }^{\ddagger}$ Glenn Hefter, ${ }^{\circledR}$ Alexander Ortner, ${ }^{\S}$ Stefan Waselikowski, ${ }^{\S}$ Markus Walther, ${ }^{\S}$ Klaas Wynne, ${ }^{\ddagger}$ and Richard Buchner, ${ }^{*} \dagger$

Institut für Physikalische und Theoretische Chemie, Universität Regensburg, D-93040 Regensburg, Germany, School of Chemistry, University of Glasgow, Glasgow, G12 8QQ

United Kingdom, Chemistry Department, Murdoch University, Murdoch, W.A. 6150, Australia, and Department of Molecular and Optical Physics, Albert-Ludwigs-Universität Freiburg, 79104 Freiburg, Germany

E-mail: Richard.Buchner@chemie.uni-regensburg.de

\footnotetext{
${ }^{*}$ To whom correspondence should be addressed

${ }^{\dagger}$ Institut für Physikalische und Theoretische Chemie, Universität Regensburg, D-93040 Regensburg, Germany

${ }^{\ddagger}$ School of Chemistry, University of Glasgow, Glasgow, G12 8QQ United Kingdom

${ }^{\top}$ Chemistry Department, Murdoch University, Murdoch, W.A. 6150, Australia

${ }^{\S}$ Department of Molecular and Optical Physics, Albert-Ludwigs-Universität Freiburg, 79104 Freiburg, Germany
} 


\begin{abstract}
Dielectric relaxation (DR) and optical Kerr-effect (OKE) spectra of the archetypal protic ionic liquids ethyl- and propylammonium nitrate (EAN \& PAN) have been measured over an unusually large frequency range from $200 \mathrm{MHz}$ to $10 \mathrm{THz}$ at temperatures (mostly) between $5{ }^{\circ} \mathrm{C}$ and $65^{\circ} \mathrm{C}$. Analysis of the low frequency $\alpha$-relaxation, associated with the cooperative relaxations of the cations (DR) and anions (OKE) and any clusters present, indicated that ion reorientation in EAN is decoupled from viscosity and occurs via cooperative relaxation involving large-angle jumps rather than rotational diffusion. Detailed consideration of the high frequency parts of the DR and OKE spectra showed that the observed intensities were a complex combination of overlapping and possibly coupled modes. In addition to previously identified intermolecular H-bond vibrations there are significant contributions from the librations of the cations and anions. The present assignments were shown to be consistent with the isotopic shifts observed for $\mathrm{d}_{3}$-EAN.
\end{abstract}

Key words: protic ionic liquids, cooperative dynamics, dielectric relaxation, optical Kerreffect, librations, isotope shift

\title{
1. Introduction
}

Protic ionic liquids (PILs) are a sub-group of room-temperature ILs that are produced via proton transfer by combination of a Brønsted acid and a Brønsted base. ${ }^{1}$ The presence of proton-donor and proton-acceptor sites is responsible for the key characteristic of PILs that distinguishes them from aprotic ILs and explains their ability to form an extended hydrogen-bond network among their cations and anions. ${ }^{2}$ Depending on the degree of proton transfer from acid to base, PILs usually have a non-negligible vapor pressure and some of them can be distilled at reduced pressure ${ }^{3}$ having boiling points below their decomposition temperatures. ${ }^{4}$ PILs have attracted particular interest as possible replacements for aqueous 
electrolyte solutions in batteries and fuel cells, where their relatively high electrical conductivity via ion or proton conduction is of particular importance. ${ }^{5,6}$ However, to tap the full potential of PILs for industrial and scientific applications, detailed knowledge of their liquid structure and their microscopic dynamics is essential.

Undoubtedly the best known PIL is ethylammonium nitrate (EAN, $\left[\mathrm{EtNH}_{3}^{+}\right]\left[\mathrm{NO}_{3}^{-}\right]$, which was first investigated almost 100 years ago by Walden. ${ }^{7}$ This salt has received considerable attention over the last few years as a model PIL due to its relatively straightforward chemical structure and also as a possible analogue for water. ${ }^{1,2,8}$ Recent studies of this important compound have included small angle neutron scattering (SANS), ${ }^{9}$ X-ray diffraction (XRD), ${ }^{10}$ and molecular dynamics (MD) simulations. ${ }^{11,12}$ Of more immediate relevance to the present investigation, Fumino et al. ${ }^{13,14}$ have reported the far-infrared (FIR) spectra of EAN and related compounds, while Weingärtner and co-workers ${ }^{15}$ have measured the dielectric spectrum of EAN up to $40 \mathrm{GHz}$ over the temperature range: $15 \leq \vartheta /{ }^{\circ} \mathrm{C} \leq 80$, subsequently extending their $25^{\circ} \mathrm{C}$ spectrum up to $2 \mathrm{THz}$ using time-domain terahertz spectroscopy (THz-TDS). ${ }^{16}$

The latter studies ${ }^{15,16}$ have established some important properties of EAN including its static permittivity or dielectric constant, $\varepsilon_{\mathrm{s}}$. The presence of low energy modes in the FIR spectrum of EAN and related compounds has attracted special interest ${ }^{13}$ because of their apparent similarity to some of the H-bond modes in water. However, unambiguous interpretation of these weak modes is not easy, particularly when using information from only one technique. For this reason Fumino et al. ${ }^{13}$ employed density functional theory (DFT) calculations to assist with their assignments. Nevertheless, a comprehensive understanding of the structure and dynamics of liquid EAN is still lacking.

The combination of ultra-fast optical Kerr-effect (OKE) and broadband dielectric relaxation (DR) spectroscopies, especially when augmented by THz-TDS and FIR measurements, has been shown to be a powerful means of investigating the structure and dynamics of imidazolium-based aprotic ILs. ${ }^{17,18}$ Accordingly, the present paper presents a detailed 
ultra-boadband study of EAN that utilizes all of these techniques. These measurements cover the exceptionally broad frequency range from $\sim 200 \mathrm{MHz}$ to $10 \mathrm{THz}$, at temperatures: $5 \leq \vartheta /{ }^{\circ} \mathrm{C} \leq 65$ and are supplemented by DR spectra of propylammonium nitrate (PAN) along with limited measurements on partially deuterated $\left(\mathrm{d}_{3^{-}}\right)$EAN at $25^{\circ} \mathrm{C}$. A preliminary discussion of some of these data has appeared ${ }^{18}$ but is significantly extended here by a more detailed quantitative analysis of the OKE and DR spectra as a whole, and in particular a comprehensive examination of the important high-frequency processes.

\section{Experimental}

\subsection{Materials}

Two batches of EAN were used. The first, which was was synthesized according to Evans $e t$ al., ${ }^{19}$ was used for all DR, OKE, viscosity and density measurements. For the conductivity measurements and deuteration a commercial sample of EAN (>97\%, Iolitec, Heilbronn, Germany) was employed. Both batches were dried under vacuum $\left(p<10^{-8}\right.$ bar $)$ for 12 days at $40{ }^{\circ} \mathrm{C}$ to minimize the water content, yielding $95 \mathrm{ppm} \mathrm{H}_{2} \mathrm{O}$ by coulometric Karl Fischer titration for the first and $20 \mathrm{ppm}$ for the second batch. For the commercial sample, halide impurities were specified by the manufacturer to be $<100 \mathrm{ppm}$. The purity of all samples was further confirmed by ${ }^{1} \mathrm{H}-\mathrm{NMR}$ spectroscopy, which showed no detectable ${ }^{1} \mathrm{H}$-containing impurities.

Partially deuterated $\mathrm{d}_{3}$-EAN was prepared by adding fresh $\mathrm{D}_{2} \mathrm{O}(99.9 \%$, Deutero $\mathrm{GmbH}$, Kastellaun, Germany) to commercial EAN and stirring for $24 \mathrm{~h}$. The solution was then evaporated under vacuum and the whole process repeated. The product so obtained was found to be $91 \%$ deuterated, corresponding to an average composition of $\left[\mathrm{EtND}_{2.7} \mathrm{H}_{0.3}\right]^{+}$. Its water content was $145 \mathrm{ppm}$.

The PAN sample was provided by QUILL at Queen's University Belfast and was prepared by dropwise addition of concentrated nitric acid to a cold solution of propylamine. The 
product was dried by lyophilisation for $24 \mathrm{~h}$ at $p=0.4$ mbar to give a pale yellow liquid. After drying for one week in high vacuum $\left(p<10^{-8}\right.$ bar $)$ at $40^{\circ} \mathrm{C}$ the water content was $420 \mathrm{ppm}$. All dried ILs were stored and handled in a nitrogen-filled glove box.

\subsection{Dielectric Spectroscopy}

Dielectric spectroscopy measures the polarization of the sample induced by an external timedependent electric field, $\vec{E}(t)$. This response is usually expressed as the frequency-dependent complex permittivity, $\hat{\varepsilon}(\nu)$ :

$$
\hat{\varepsilon}(\nu)=\varepsilon^{\prime}(\nu)-\mathrm{i} \varepsilon^{\prime \prime}(\nu)
$$

and contains contributions from rotational, vibrational and translational motions. The relative permittivity, $\varepsilon^{\prime}(\nu)$, shows a dispersion from the static permittivity, $\varepsilon_{\mathrm{s}}=\lim _{\nu \rightarrow 0} \varepsilon^{\prime}(\nu)$ to the permittivity at infinite frequency, $\varepsilon_{\infty}=\lim _{\nu \rightarrow \infty} \varepsilon^{\prime}(\nu)$. The dielectric loss, $\varepsilon^{\prime \prime}(\nu)$, describes the dissipation of energy within the sample arising from the coupling of $\vec{E}(t)$ to dipole fluctuations. Dielectric measurements were performed using an Agilent E8364B Vector Network Analyzer (VNA) combined with two reflection probes (85070E-020 and -050) operating in the frequency ranges $0.2 \leq \nu / \mathrm{GHz} \leq 20$ and $1 \leq \nu / \mathrm{GHz} \leq 50$, respectively, ${ }^{20}$ and a waveguide interferometer covering the frequencies $60 \leq \nu / \mathrm{GHz} \leq 89 .{ }^{21}$ Air, mercury and $N, N$-dimethylacetamide were used respectively as open, short and load standards for the calibration of the VNA setup. Raw VNA data were corrected for calibration errors with a Padé approximation using purified benzonitrile and 1-butanol as secondary calibration standards. ${ }^{22}$ VNA data were cross-checked in the range $27 \leq \nu / \mathrm{GHz} \leq 40$ using a waveguide transmission cell mounted to the VNA. All DRS experiments were carried out from $5{ }^{\circ} \mathrm{C}$ to $65^{\circ} \mathrm{C}$ in $10^{\circ} \mathrm{C}$ steps, with an overall temperature stability and accuracy of $\pm 0.05^{\circ} \mathrm{C}$. For PAN, additional low frequency $(0.05-0.5 \mathrm{GHz})$ dielectric measurements were performed at 5 , 15 and $25^{\circ} \mathrm{C}$ using the cut-off type reflection cell described elsewhere. ${ }^{21}$

Data in the terahertz region were collected using a transmission/reflection time-domain 
terahertz spectrometer (THz-TDS ${ }^{23}$ (at Freiburg), a Bruker Vertex 70 FTIR spectrometer ${ }^{17}$ for EAN and a Bruker IFS 66v FTIR for PAN (at Glasgow). The THz-TDS instrument covered the frequency range $0.3 \leq \nu / \mathrm{THz} \leq 3$ and the others $0.9 \leq \nu / \mathrm{THz} \leq 12$, respectively. Far-infrared (FIR) absorbance data were converted to complex permittivities using the Kramers-Kronig relation as described elsewhere. ${ }^{24}$ Both THz and FIR spectra were measured from $5{ }^{\circ} \mathrm{C}$ to $65^{\circ} \mathrm{C}$ in $20^{\circ} \mathrm{C}$ steps with a temperature stability of $\pm 0.5^{\circ} \mathrm{C}$. Combination of all these data provided dielectric spectra (Figs. 3 and 4) covering the frequency range $0.2 \leq \nu / \mathrm{GHz} \leq 10000$. Additionally, THz-TDS and FTIR measurements of $\mathrm{d}_{3}$-EAN were performed at $25^{\circ} \mathrm{C}$ between $25 \mathrm{~cm}^{-1}$ and $450 \mathrm{~cm}^{-1}(0.7 \lesssim \nu / \mathrm{THz} \lesssim 13)$.

For electrically conducting samples like ILs, it is convenient for the data analysis to remove the contribution to the measured spectra due to the steady-state movement (the direct current, dc) of ions in the applied electric field. This is achieved by subtracting the Ohmic loss from the experimentally-accessible total loss, $\eta^{\prime \prime}(\nu)$ :

$$
\varepsilon^{\prime \prime}(\nu)=\eta^{\prime \prime}(\nu)-\frac{\kappa}{2 \pi \nu \varepsilon_{0}},
$$

where $\kappa$ is the dc conductivity and $\varepsilon_{0}$ is the permittivity of free space. ${ }^{25,26}$

Conductivity corrections can be determined either by direct measurement at low frequencies or by treating $\kappa$ as an additional adjustable parameter in the fitting procedure. Due to fringing field effects caused by the geometry of the VNA probes, fitted conductivities differ slightly from experimental values. Thus, measured $\kappa$ values were used as a starting approximation for the conductivity correction but were adjusted to obtain the best fit. The corrected conductivities differed from the experimental values by $\sim 2-16 \%$.

\subsection{OKE Spectroscopy}

In optical Kerr-effect spectroscopy the dynamics of the polarizability anisotropy response of the system are observed. This response is represented by the time-derivative of the two-point 
time-correlation function of the anisotropic part of the many-body polarizability tensor, $\Pi:{ }^{27}$

$$
S_{\mathrm{OKE}}(t) \propto \frac{1}{k_{\mathrm{B}} T} \frac{\mathrm{d}}{\mathrm{d} t}\left\langle\Pi_{x y}(t) \Pi_{x y}(0)\right\rangle
$$

Two OKE setups were employed to capture the full time span of the orientational and intermolecular dynamics of EAN. For the faster dynamics a Coherent Mira-SEED oscillator was used that generated $800 \mathrm{~nm}$ pulses with an energy of $8 \mathrm{~nJ}$ and a pulse duration of $20 \mathrm{fs}$ at a repetition rate of $76 \mathrm{MHz}$. The parent beam was split into $80 \%$-pump and $20 \%$ probe beams which were co-focused into a quartz cuvette containing the sample. For lower temperatures the sample was controlled to $\pm 0.1^{\circ} \mathrm{C}$ by a cryostat (Oxford Instruments, DN). Above room temperature a home-built copper heating block with a temperature stability of $\pm 0.1{ }^{\circ} \mathrm{C}$ was employed. A $600 \mathrm{~mm}$ delay line, with a resolution of $50 \mathrm{~nm}(0.33 \mathrm{fs})$ gave a time scale of $4 \mathrm{~ns}$, resulting in a bandwidth of $c a .250 \mathrm{MHz} .{ }^{17}$ For slower dynamics a similar setup using a regeneratively-amplified laser (Coherent Legend USX) with a stretched pulse duration of $c a$. 1 ps was used to increase the signal-to-noise ratio of the weak relaxation signal at longer times. The two time-domain signals were concatenated, Fourier-transformed to the frequency domain, and deconvoluted from the instantaneous response, ${ }^{18}$ to yield the final OKE spectra.

\subsection{Physical Properties}

Densities, $\rho$, were measured from $15^{\circ} \mathrm{C}(\mathrm{EAN})$ or $5{ }^{\circ} \mathrm{C}(\mathrm{PAN})$ to $65^{\circ} \mathrm{C}$ in $10{ }^{\circ} \mathrm{C}$ steps using a vibrating-tube densimeter (Anton Paar, Graz, Austria, DMA 5000 M) with a temperature stability of $\pm 0.01^{\circ} \mathrm{C}$ and a precision of $\pm 5 \cdot 10^{-6} \mathrm{~g} \mathrm{~cm}^{-3}$ using the calibration supplied by the manufacturer. Viscosities, $\eta$, were determined with a reproducibility of $\sim 0.5 \%$ using an automated rolling ball microviscometer (Anton Paar, Graz, Austria, AMVn). These measurements covered temperatures from $5{ }^{\circ} \mathrm{C}$ to $125^{\circ} \mathrm{C}$ in $10^{\circ} \mathrm{C}$ steps with an uncertainty of $\pm 0.05^{\circ} \mathrm{C}$. Electrical conductivities, $\kappa$, of EAN were measured between $-5^{\circ} \mathrm{C}$ and $125^{\circ} \mathrm{C}$ 
$\left( \pm 0.01{ }^{\circ} \mathrm{C}\right)$ in $10{ }^{\circ} \mathrm{C}$ steps using capillary cells in conjunction with a programmable precision

LCR meter (Hameg, Mainhausen, Germany, HM8118) as described elsewhere. ${ }^{28}$ The overall relative uncertainty in $\kappa$ was estimated to be $\pm 0.5 \%$. Due to material constraints, none of the physicochemical properties of $\mathrm{d}_{3}$-EAN, nor $\kappa(\mathrm{PAN})$ were measured.

\section{Results}

\subsection{Densities, Viscosities and Conductivities}

The data obtained for $\rho$ and $\eta$ for EAN and PAN and $\kappa($ EAN $)$ as functions of temperature, $T$, are given in Table S1 of the Supplementary Information (SI). The densities followed the linear relations

$$
\begin{array}{ll}
\text { EAN }: & \rho(T / K)=1.39072-6.05056 \cdot 10^{-4} \cdot T / K \\
\text { PAN }: & \rho(T / K)=1.32833-5.95470 \cdot 10^{-4} \cdot T / K
\end{array}
$$

over the temperature range investigated. The EAN results are in good agreement $(\sim 0.1 \%)$ with the data of Weingärtner et al. ${ }^{15}$ but differ by $\sim 0.5 \%$ and $\sim 0.7 \%$, respectively, from those of Greaves et al. ${ }^{29}$ and Poole et al. ${ }^{30,31}$ For $\rho(\mathrm{PAN})$ at $25^{\circ} \mathrm{C}$, the only temperature where comparison is possible, the present value is in only modest agreement with the results of Greaves et al. ${ }^{1}(\sim 0.5 \%)$ and Atkin et al. ${ }^{32}(\sim 0.8 \%)$.

The effects of temperature on $\eta$ (and for EAN, $\kappa$, expressed as the resistivity, $\kappa^{-1}$ ) are displayed in logarithmic form in Fig. 1. The present viscosities of EAN are systematically higher than those reported in the literature. At $25^{\circ} \mathrm{C}$, where the most extensive comparison can be made, the literature values are $34.9 \mathrm{mPas},{ }^{15} 26.9 \mathrm{mPas},{ }^{30} 32.1 \mathrm{mPas}^{31}$ and $36 \mathrm{mPas},{ }^{33}$ compared with the present result of $38.6 \mathrm{mPas}$ (Table $\mathrm{S} 1$ ). This may reflect a lower water content in our material. Consistent with this inference, the temperature dependence of the present viscosities is almost identical (Fig. 1a) to that of Weingärtner et 
$a l .{ }^{15}$ The present electrical conductivities of EAN are in reasonable agreement $(\sim 3 \%)$ with the values reported (over a much smaller temperature range) by Oleinikova et al. ${ }^{34}$ (Fig. $1 b)$.
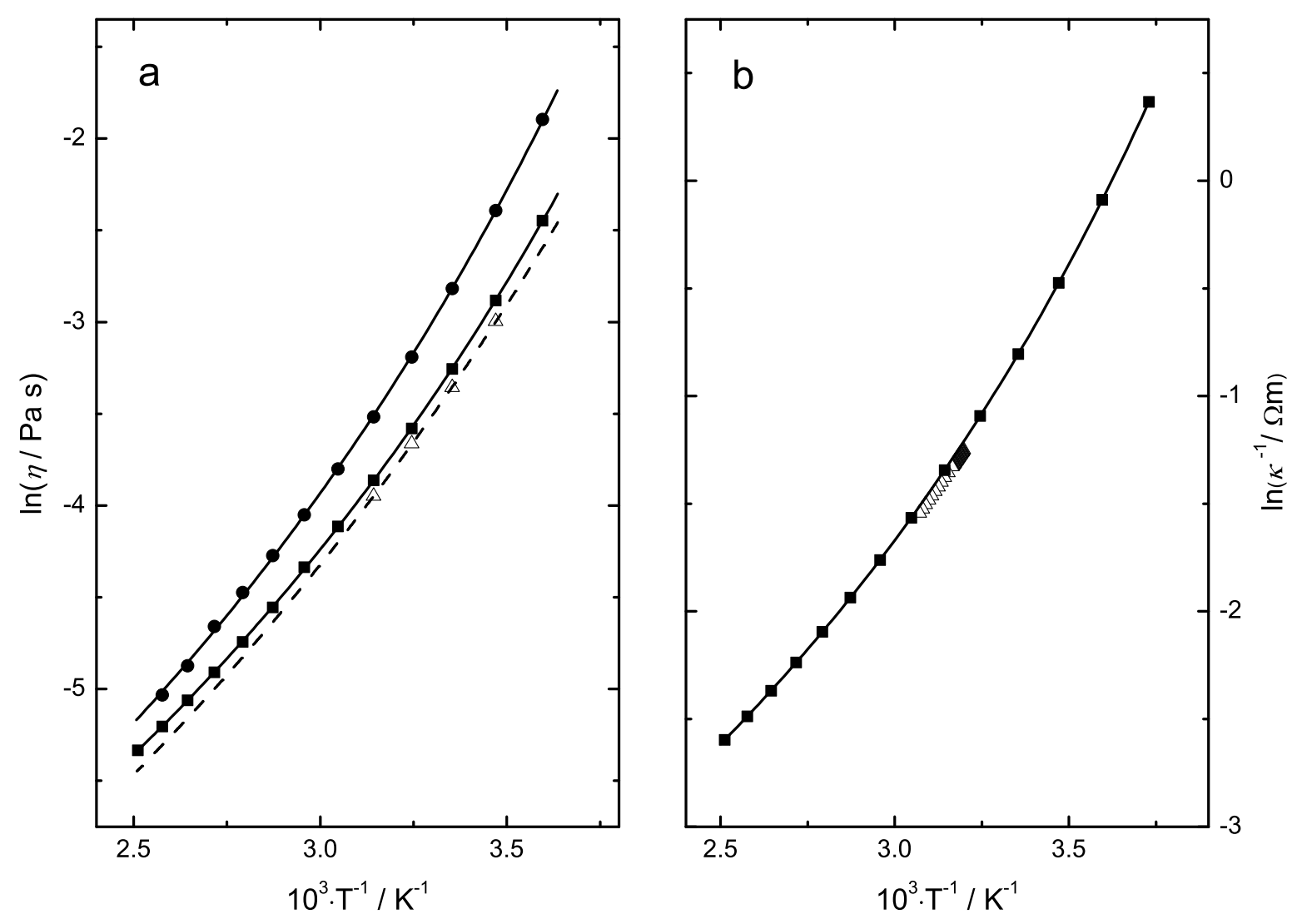

Figure 1: Present results for the physical properties of EAN (ם) and PAN (-) as functions of temperature, $T$ : (a) viscosity, $\eta$, and (b) electrical resistivity, $\kappa^{-1}$. Also included are literature data ${ }^{15,34}(\triangle)$ and fits with eq 6 (lines).

The viscosities of EAN and PAN and the electrical conductivities of EAN follow the Vogel-Fulcher-Tammann (VFT) equation ${ }^{35}$

$$
\ln Y=\ln Y_{0}+\frac{B_{\mathrm{VFT}}}{T-T_{0}}
$$

where $Y=\eta$ or $\kappa^{-1}$, which is commonly used to describe the temperature dependence of transport properties of glass-forming liquids above their glass-transition temperature, $T_{\mathrm{g}} .{ }^{35,36}$ In eq $6, Y_{0}, B_{\mathrm{VFT}}$ and $T_{0}$ (the so-called Vogel temperature) are fitting parameters. The fit 
results are listed in Table S2 along with corresponding literature data. ${ }^{15,34}$ The present values of the so-called fragility parameter, $B_{\mathrm{VFT}}$, of EAN are in only modest agreement with those of Weingärtner et al. ${ }^{15}$ and Oleinikova et al. ${ }^{34}$ especially for the viscosities, but the $T_{0}$ values agree well. As $T_{0}$ is usually about $30 \mathrm{~K}$ below, $T_{\mathrm{g}},{ }^{36}$ the present value of $T_{0}=147.65 \mathrm{~K}$ for EAN is also consistent with the result of $T_{\mathrm{g}}=181.65 \mathrm{~K}$ reported by Belieres et al. ${ }^{33}$ The present VFT parameters of PAN are broadly compatible with those of Smith et al. ${ }^{37}$ but less so with those of Bouzón Capelo et al. ${ }^{38}$

\subsection{Spectroscopic Model Selection and Fitting}

General comments. Representative OKE and DR spectra of EAN and DR spectra of PAN over the frequency range: $200 \mathrm{MHz} \leq \nu \leq 10 \mathrm{THz}$ at temperatures from (5 to 65$)^{\circ} \mathrm{C}$ are presented in Figs. 2, 3 and 4, respectively. OKE spectra of PAN are reported elsewhere. ${ }^{18}$ It should be stressed that the dielectric spectra were resampled (i.e. linearly interpolated) prior to fitting. This was necessary to achieve an equal point density over the entire frequency range and to stabilize the fit of the small intensities at high frequencies.

To understand the spectra on a molecular level an appropriate mathematical description of the experimental data is necessary. This was achieved by fitting $\hat{\varepsilon}(\nu)$ or the frequencydependent OKE response, $S_{\mathrm{OKE}}(\nu)$, with a sum of $n$ individual processes of band-shape function $\tilde{F}_{j}(\nu)$ and amplitude $S_{j}$ :

$$
y=\sum_{j=1}^{n} S_{j} \cdot \tilde{F}_{j}(\nu)+y_{\infty}
$$

where $y=\hat{\varepsilon}(\nu) \& y_{\infty}=\varepsilon_{\infty}$ for DR and $y=S_{\mathrm{OKE}}(\nu) \& y_{\infty}=0$ for OKE spectra. Lowerfrequency modes $(\lesssim 100 \mathrm{GHz})$ associated with molecular reorientations were modelled using relaxation functions, whereas librations and intermolecular vibrations in the terahertz region were better modelled as resonances. ${ }^{25}$ However, before describing the model ultimately selected it is important to recognize that fitting DR and OKE spectra over such a broad 


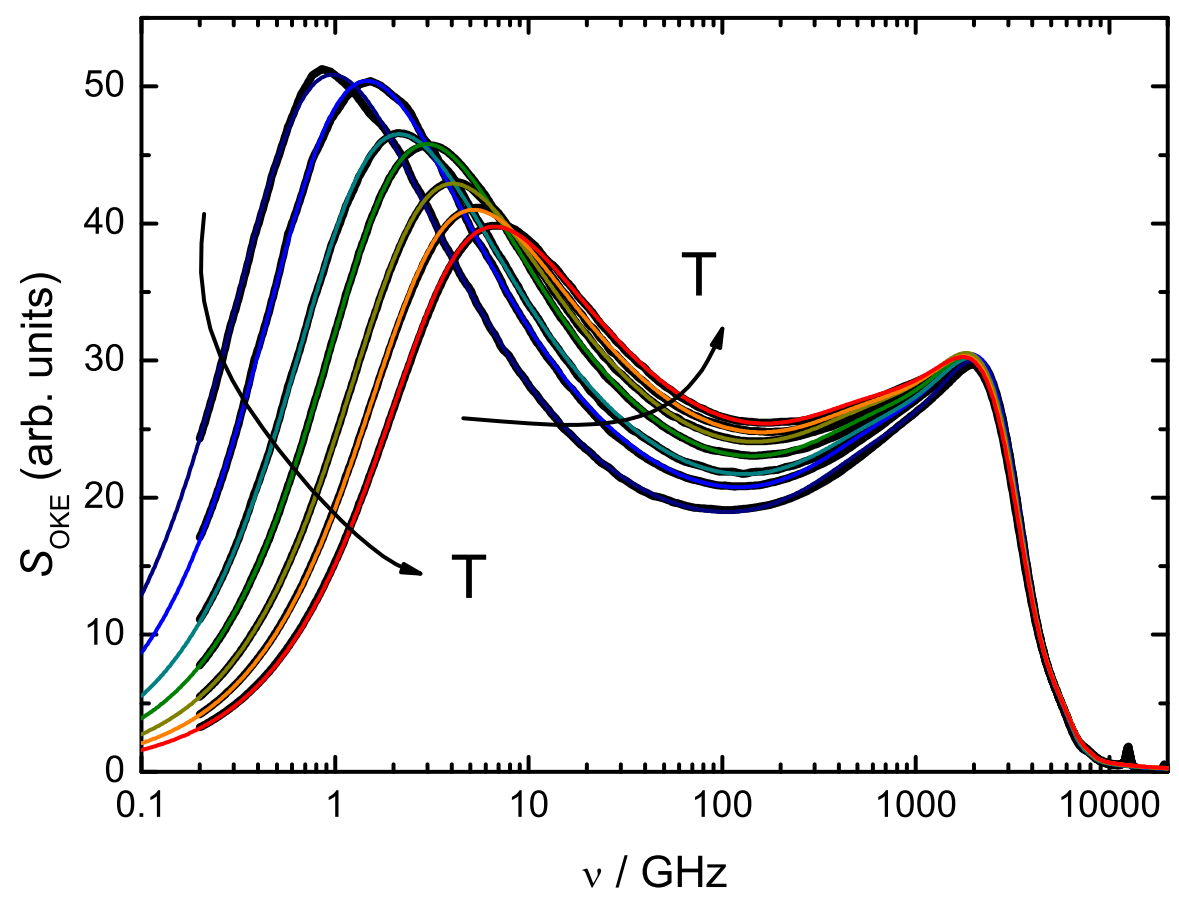

Figure 2: Optical Kerr-effect spectra of EAN from $5{ }^{\circ} \mathrm{C}$ to $65^{\circ} \mathrm{C}$ in $10{ }^{\circ} \mathrm{C}$ steps. Black circles are experimental data; overlaying colored lines represent the total fit.

frequency range is a formidable task that cannot be performed $a b$ initio. It therefore follows that any mathematical description adopted invariably involves some degree of choice. Because the fitting of the spectra is critical to their interpretation, it is appropriate to discuss this aspect of this work in some detail.

Difficulties in fitting OKE and DR spectra. The major difficulties in fitting the present spectra can be summarized as follows. First, resonance and (particularly) relaxation modes are often broad and generally strongly overlapping. Second, there are certain theoretical and computational problems associated with some of the standard mathematical models used to describe the modes (see below). Third, the present (in particular the DR) spectra exhibit a broad featureless and low (but finite) intensity over the intermediate frequency range $0.05 \lesssim \nu / \mathrm{THz} \lesssim 1$ (Figs. $2-6$ ), which is particularly hard to model. Fourth, the accuracy of the spectra is limited by the unwanted conductivity contribution (eq2), which swamps the DR (but not the OKE) signal at low frequencies (say, $\nu \lesssim 0.5 \mathrm{GHz}$ ), and by 
current technological limitations on the accuracy of the apparatus at higher frequencies (say, $\nu \gtrsim 100 \mathrm{GHz}$ ). Lastly, there are no a priori guidelines that limit the number or nature of the modes present; this is especially critical because the modes are expected to be strongly coupled and overlapping. ${ }^{39}$ The main reason is that DRS and OKE spectroscopy both probe collective dynamics and thus their spectra not only arise from the motions of individual molecules or ions but also reflect coupling effects. In ionic liquids not only the motions of the various species are coupled but also translational and rotational motions are not independent from each other. In particular for the rotation-translation cross-correlations it is well known from simulations that they may yield negative contributions to IL spectra. ${ }^{40-43}$ Unfortunately, until now computer simulations are only able to reach — at best - qualitative agreement with experimental DR and OKE spectra. ${ }^{44}$ For this reason we believe that the classical spectroscopist's approach, based on eq 7 and described in detail below, is still useful.

For relaxation processes, which usually appear at lower frequencies $(\lesssim 100 \mathrm{GHz})$, the empirical Havriliak-Negami (HN) equation of band shape $\tilde{F}_{\mathrm{HN}}$ is suitable. For reasons described in detail elsewhere, ${ }^{18,45}$ the $\mathrm{HN}$ equation was used in its inertia-corrected form (HNi):

$$
\tilde{F}_{\mathrm{HNi}}(\nu)=\left(S_{\mathrm{HNi}}^{0}\right)^{-1}\left[\frac{1}{\left(1+(\mathrm{i} 2 \pi \nu \tau)^{1-\alpha}\right)^{\beta}}-\frac{1}{\left(1+\left(\mathrm{i} 2 \pi \nu \tau+\gamma_{\mathrm{lib}} \tau\right)^{1-\alpha}\right)^{\beta}}\right]
$$

where $\tau$ is the relaxation time, $\gamma_{\text {lib }}$ the inertial rise rate, $\alpha$ and $\beta$ the width parameters and $S_{\mathrm{HNi}}^{0}$ the amplitude normalization factor

$$
S_{\mathrm{HNi}}^{0}=1-\frac{1}{\left(1+\left(\gamma_{\mathrm{lib}} \tau\right)^{1-\alpha}\right)^{\beta}}
$$

The limiting forms of the Havriliak-Negami equation are the Cole-Davidson $\left(\mathrm{CD}, \alpha_{j}=0\right)$, Cole-Cole $\left(\mathrm{CC}, \beta_{j}=1\right)$ and Debye $\left(\mathrm{D}, \alpha_{j}=0, \beta_{j}=1\right)$ equations and their inertia-corrected versions $\mathrm{CDi}, \mathrm{CCi}$ and Di. ${ }^{45}$

The higher-frequency (terahertz and FIR) region is dominated mainly by librational 
motions and low-energy inter- and intra-molecular vibrations. Such processes are best represented by a resonance-type band-shape function such as a damped harmonic (Brownian) oscillator $(\mathrm{DHO})^{46}$

$$
\tilde{F}_{\mathrm{DHO}}(\nu)=\frac{\nu_{0}^{2}}{\left(\nu_{0}^{2}-\nu^{2}\right)+\mathrm{i} \nu \gamma_{\mathrm{DHO}}}
$$

with resonance frequency, $\nu_{0}$, and damping constant, $\gamma_{\mathrm{DHO}}$. Alternatively, an antisymmetrized Gaussian oscillator $(\mathrm{G})$,

$$
\begin{aligned}
\tilde{F}_{\mathrm{G}}(\nu) & =\left(S_{\mathrm{G}}^{0}\right)^{-1}\left[\frac{2}{\sqrt{\pi}} \times \mathrm{Da}\left(\frac{\nu+\nu_{0}}{\sqrt{2} \gamma_{\mathrm{G}}}\right)-\frac{2}{\sqrt{\pi}} \times \mathrm{Da}\left(\frac{\nu-\nu_{0}}{\sqrt{2} \gamma_{\mathrm{G}}}\right)\right. \\
& \left.+\mathrm{i}\left(\exp \left[-\frac{\left(\nu-\nu_{0}\right)^{2}}{2 \gamma_{\mathrm{G}}^{2}}\right]-\exp \left[-\frac{\left(\nu+\nu_{0}\right)^{2}}{2 \gamma_{\mathrm{G}}^{2}}\right]\right)\right]
\end{aligned}
$$

with resonance frequency, $\nu_{0}$, and half bandwidth, $\gamma_{\mathrm{G}}$, can be used where

$$
S_{\mathrm{G}}^{0}=\frac{4}{\sqrt{\pi}} \times \mathrm{Da}\left(\frac{\nu_{0}}{\sqrt{2} \gamma_{\mathrm{G}}}\right)
$$

In eqs $11 \& 12, \mathrm{Da}(x)$ is Dawson's integral ${ }^{47}$ and $S_{\mathrm{G}}^{0}$ is the normalization factor for this band-shape function.

Adopted fitting approach. Bearing in mind the difficulties discussed above, after an extensive investigation of the possibilities, the following description (presented as five dot points) was adopted for the OKE and DR spectra of EAN. As will be shown, this description was also found to be applicable without modification to PAN (Section 5.3) and $\mathrm{d}_{3}$-EAN (Section 5.2).

- The broad-band OKE and DR spectra of EAN at all temperatures (Figs. 2 and 3) show two clear features: a dominant temperature-dependent relaxation in the low $\mathrm{GHz}$ region and a much narrower almost temperature-independent resonance process in the $\mathrm{THz}$ region. The dominant low $\mathrm{GHz}$ process is the so-called $\alpha$-relaxation, ${ }^{18,48}$ which was found to be best described by an inertia-corrected Cole-Davidson (CDi) 

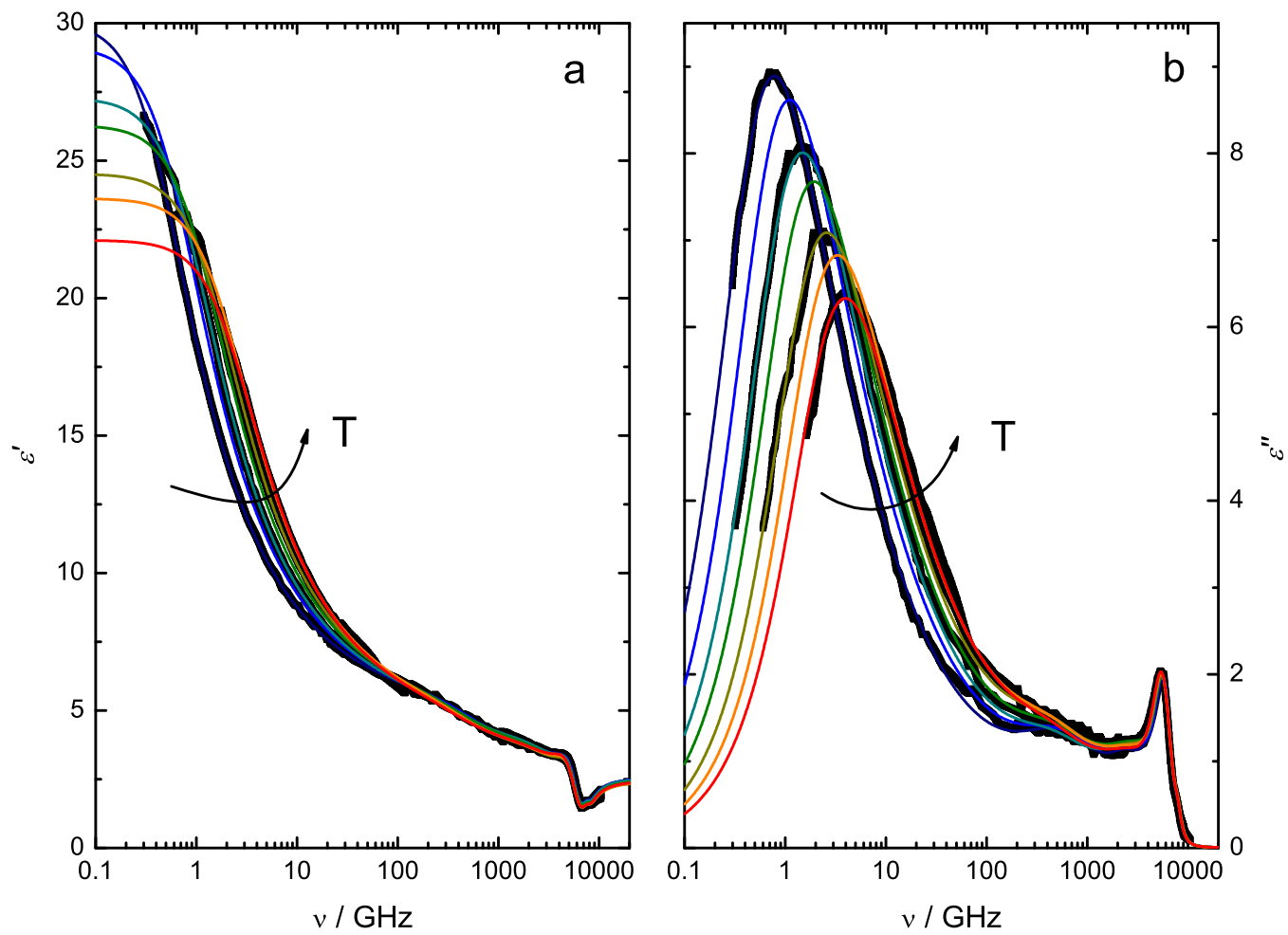

Figure 3: (a) Permittivity, $\varepsilon^{\prime}(\nu)$, and (b) dielectric loss, $\varepsilon^{\prime \prime}(\nu)$, spectra of EAN from $5{ }^{\circ} \mathrm{C}$ to $65^{\circ} \mathrm{C}$ in $10^{\circ} \mathrm{C}$ steps. For visual clarity data at 15,35 , and $55^{\circ} \mathrm{C}$ are not displayed. Black squares are resampled experimental data; overlaying colored lines represent the overall fit.

function (eq 8 with $\alpha_{\mathrm{CDi}}=0$ ). For stabilization of the fits of the DR spectra the width parameter, $\beta$, was fixed at 0.5 for all temperatures, as it varied only slightly with $T$ when allowed to float. Note that an asymmetrical (i.e., a CD) band shape, broadened to higher frequencies, was preferred over the symmetrically-broadened (CC) function used for other ILs, ${ }^{48}$ because the low frequency wing of the $\alpha$-mode of EAN exhibited a Debye, rather than a broadened, band shape (Fig. 5). The high frequency (inertial) termination of the $\alpha$-relaxation was achieved by fixing $\gamma_{\text {lib }}$ at $3 \mathrm{THz}$ and $5 \mathrm{THz}$ for the fits of the OKE and DR spectra, respectively.

- As discussed elsewhere, ${ }^{18}$ the featureless intensity of the OKE spectrum in the intermediate frequency range $50 \lesssim \nu / \mathrm{GHz} \lesssim 1000$ (i.e., "intermediate" between the $\alpha$-relaxation and the higher frequency librational modes) was approximated by a modified Cole-Cole function (CCm). In the limit of $\alpha_{\mathrm{CCm}} \rightarrow 1$ this contribution appears as 

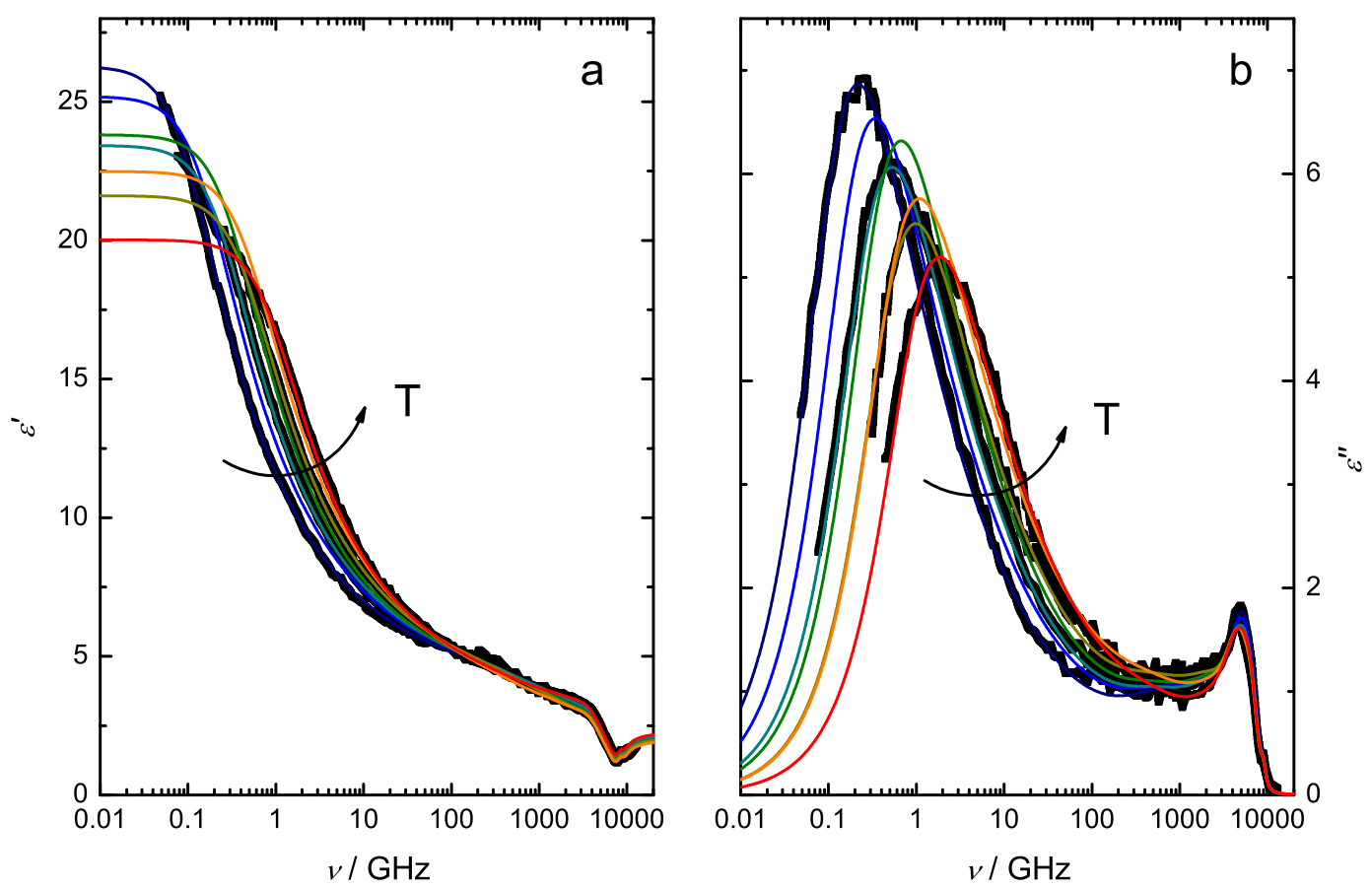

Figure 4: (a) Permittivity, $\varepsilon^{\prime}(\nu)$, and (b) dielectric loss, $\varepsilon^{\prime \prime}(\nu)$, spectra of PAN from $5{ }^{\circ} \mathrm{C}$ to $65^{\circ} \mathrm{C}$ in $10^{\circ} \mathrm{C}$ steps. For visual clarity data at 15,35 , and $55^{\circ} \mathrm{C}$ are not displayed. Black squares are resampled experimental data; overlaying colored lines represent the overall fit.

a constant loss (CL), ${ }^{18}$ which corresponds to a logarithmic decay in the time domain, which is typical for glass-forming liquids. ${ }^{49}$ The CL contribution was made compatible with the spectroscopic dynamics in the intermediate region by the application of an inertial correction at high frequencies and the so-called $\alpha$-termination at low frequencies. ${ }^{18}$ This CL "mode" is undoubtedly a composite but little can be said at this stage about the nature of the processes that produce it or their relative contributions.

In the course of re-analyzing the high frequency parts of the OKE and DR spectra it was found necessary to modify slightly the previous ${ }^{18}$ fit model. In doing so, it turned out that the CL contribution, which is indispensable for describing the OKE spectra ${ }^{18}$ can be neglected in the DR spectra. This is because the amplitude of this contribution is much smaller in the latter; in addition, inclusion of a CL contribution did not improve the fit either in the rather poorly defined region near $100 \mathrm{GHz}$ or overall in terms of the normalized variance of the fit, $\chi_{\mathrm{r}}^{2}$. Furthermore, application 
of the CL term to the DR spectra of PAN produced physically impossible negative values of the CL amplitude, $S_{\mathrm{CL}}$. These findings do not prove the absence of a CL contribution to the DR spectra; more likely it is subsumed in the neighboring CDi and $\mathrm{DHO}_{1}$ modes. Removal of the CL contribution in all the DR spectra reduced the number of fitting parameters without affecting $\chi_{\mathrm{r}}^{2}$, consistent with the usual criteria for model selection. ${ }^{50}$

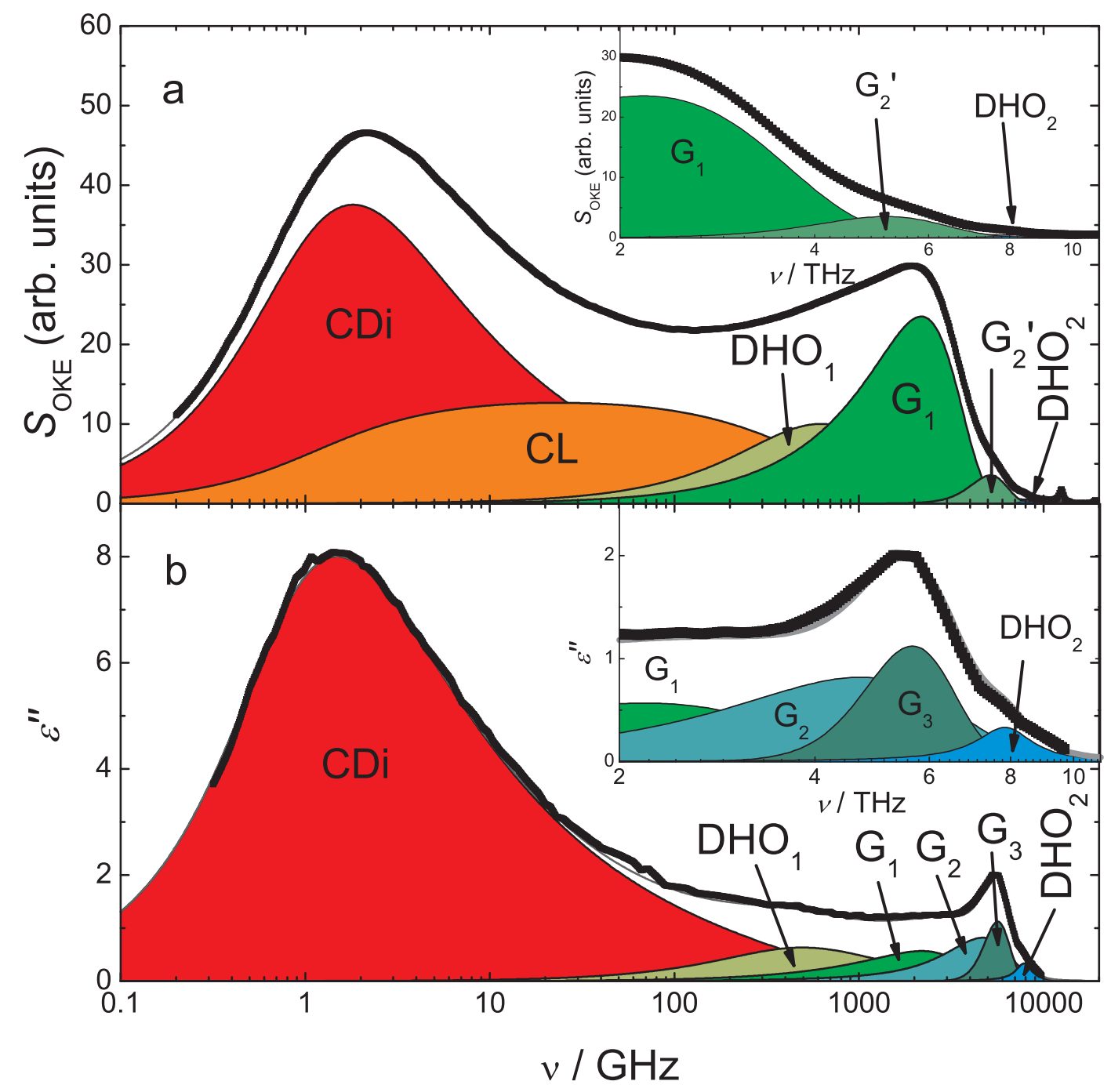

Figure 5: The imaginary part of (a) OKE and (b) DR spectra of EAN at $25^{\circ} \mathrm{C}$. Black squares are resampled experimental data and thin gray lines represent the overall fit. Shaded areas indicate the contributions of the individual processes assuming a $\left(\mathrm{CDi}+\mathrm{CL}+\mathrm{DHO}_{1}+\mathrm{G}_{1}\right.$ $\left.+\mathrm{G}_{2}^{\prime}+\mathrm{DHO}_{2}\right)$ model for the OKE spectrum and a $\left(\mathrm{CDi}+\mathrm{DHO}_{1}+\mathrm{G}_{1}+\mathrm{G}_{2}+\mathrm{G}_{3}+\right.$ $\mathrm{DHO}_{2}$ ) model for the DR spectrum. Insets: expanded views of the high frequency data. 
- While there was no explicit indication of a mode at $\sim 1 \mathrm{THz}$ in the present spectra of EAN (Figs. 2 and 3), OKE measurements at low $T$ clearly indicated the existence of such a mode for PAN. ${ }^{18}$ Accordingly, an analogous mode, $\mathrm{DHO}_{1}$, was assumed for EAN, which considerably improved $\chi_{\mathrm{r}}^{2}$.

- At $\nu>1 \mathrm{THz}$, both OKE and DR spectra of EAN were dominated by apparent modes at $\sim 2 \mathrm{THz}$ (Figs. 2 and $5 \mathrm{a}$ ) and $\sim 5.5 \mathrm{THz}$ (Figs. 3 and 5b), respectively. To account for the observed intensities in this frequency range Gaussians were preferred over DHOs as they better described the steep decay of the OKE and $\varepsilon^{\prime \prime}(\nu)$ intensities at $5.5 \lesssim \nu / \mathrm{THz} \lesssim 7.2$. It was further established that the intensity in this region of the OKE spectra was best described by a large-amplitude Gaussian, $\mathrm{G}_{1}$, centered at $\sim 2 \mathrm{THz}$ and a second weaker Gaussian, $\mathrm{G}_{2}^{\prime}$, that accounted for the poorly defined shoulder at $\sim 5 \mathrm{THz}$ (Figs. 2 and $5 \mathrm{a}$ ). For the DR spectra (Fig. 5b), the $\mathrm{G}_{1}$ mode was again required; however, while a $\mathrm{G}_{2}^{\prime}$ mode at $\sim 5.5 \mathrm{THz}$ provided reasonable fits, ${ }^{18}$ considerable improvement was achieved by splitting it into two Gaussians, $\mathrm{G}_{2}$ and $\mathrm{G}_{3}$. Support for the existence of an additional process comes from the DR spectra of PAN, which show a small but distinct shoulder at $\sim 7.5 \mathrm{THz}$ (Fig. 6 inset). These findings in turn suggest that the $\mathrm{G}_{2}^{\prime}$ mode in the OKE spectra (Fig. 5a) is almost certainly a composite, consistent with its resonance frequency lying between those of $\mathrm{G}_{2}$ and $\mathrm{G}_{3}$ in the DR spectra.

- To account for the remaining intensity at the highest frequencies in both DR and OKE spectra, that is, a poorly defined shoulder in EAN (Fig. 5 insets) and a small peak in the PAN spectrum (Fig. 6 inset), it was necessary to include a mode, $\mathrm{DHO}_{2}$, corresponding to the intramolecular mode reported by Fumino et al. ${ }^{13}$ at $7.9 \mathrm{THz}\left(263.3 \mathrm{~cm}^{-1}\right)$ in the FIR spectrum of EAN. The resonance frequency of this mode was fixed at $7.9 \mathrm{THz}$ and its amplitude, $S_{\mathrm{DHO} 2}$, and damping constant, $\gamma_{\mathrm{DHO} 2}$, were arbitrarily set to the values listed in Tables SI3 and SI4 to achieve convergence. 
Thus the models adopted as providing the best description of the spectra over the extraordinarily-wide range of frequencies used in this study were, for OKE: (CDi + CL $\left.+\mathrm{DHO}_{1}+\mathrm{G}_{1}+\mathrm{G}_{2}^{\prime}+\mathrm{DHO}_{2}\right)$ and for DR: $\left(\mathrm{CDi}+\mathrm{DHO}_{1}+\mathrm{G}_{1}+\mathrm{G}_{2}+\mathrm{G}_{3}+\mathrm{DHO}_{2}\right)$. The overall fits obtained at $25^{\circ} \mathrm{C}$ are shown in Fig. 5a (OKE) and Fig. 5b (DR). Similar fits (not shown) were obtained for the OKE and DR spectra at 5,45 and $65^{\circ} \mathrm{C}$ and the parameters so derived are summarized in Tables SI3 \& SI4. Note however that, due to the absence of THz-TDS and FIR data at 15,35 and $55^{\circ} \mathrm{C}$, parameters also listed for convenience in Tables SI3 \& SI4 for the resonance modes $\left(\mathrm{DHO}_{1}, \mathrm{G}_{1}, \mathrm{G}_{2} \& \mathrm{G}_{3}\right)$ at these temperatures were obtained by linear interpolation.

Fitting of $\mathrm{d}_{3}$-EAN and PAN. The DR and OKE spectra of $\mathrm{d}_{3}$-EAN at $25^{\circ} \mathrm{C}$ were also fitted with the models used for EAN with fixed $\gamma_{\text {lib }}=5 \mathrm{THz}$ and $\nu_{0, \mathrm{DHO} 2}=7.7 \mathrm{THz}$ (Fig. S1). For the DR spectra of PAN the mathematical model used for EAN also provided a fully satisfactory fit at the temperatures studied (Fig. 6). The derived parameters are listed in Table S5. As for EAN the parameters of the resonance modes $\left(\mathrm{DHO}_{1}, \mathrm{G}_{1}, \mathrm{G}_{2} \& \mathrm{G}_{3}\right)$ of PAN at 15,35 and $55^{\circ} \mathrm{C}$ were obtained by linear interpolation. The resonance frequency of the $\mathrm{DHO}_{2}$ mode, $\nu_{0, \mathrm{DHO}}$, in $\mathrm{PAN}$ was fixed at $9.0 \mathrm{THz}$ consistent with that reported by Fumino et al. ${ }^{13}$ and its amplitude, $S_{\mathrm{DHO} 2}$, and damping constant, $\gamma_{\mathrm{DHO} 2}$, were set arbitrarily to the values listed in Table S5 to achieve convergence. The inertial rise rate, $\gamma_{\text {lib }}$, was fixed at $5 \mathrm{THz}$. For a discussion of the OKE spectra of PAN the reader is referred to ref. 51.

Comparison of the DR spectra of EAN and PAN (Figs. 3 and 4) revealed that the latter were significantly more scattered over the entire frequency range. In particular, the increased noise in the THz-TDS data exacerbated an unambiguous concatenation of the FIR and lower frequency spectra. This introduced additional uncertainties into the fit of the intermediate and high frequency regions of the PAN spectra; nevertheless, the fit parameters so obtained are broadly consistent with the EAN data (Section 5.3). 


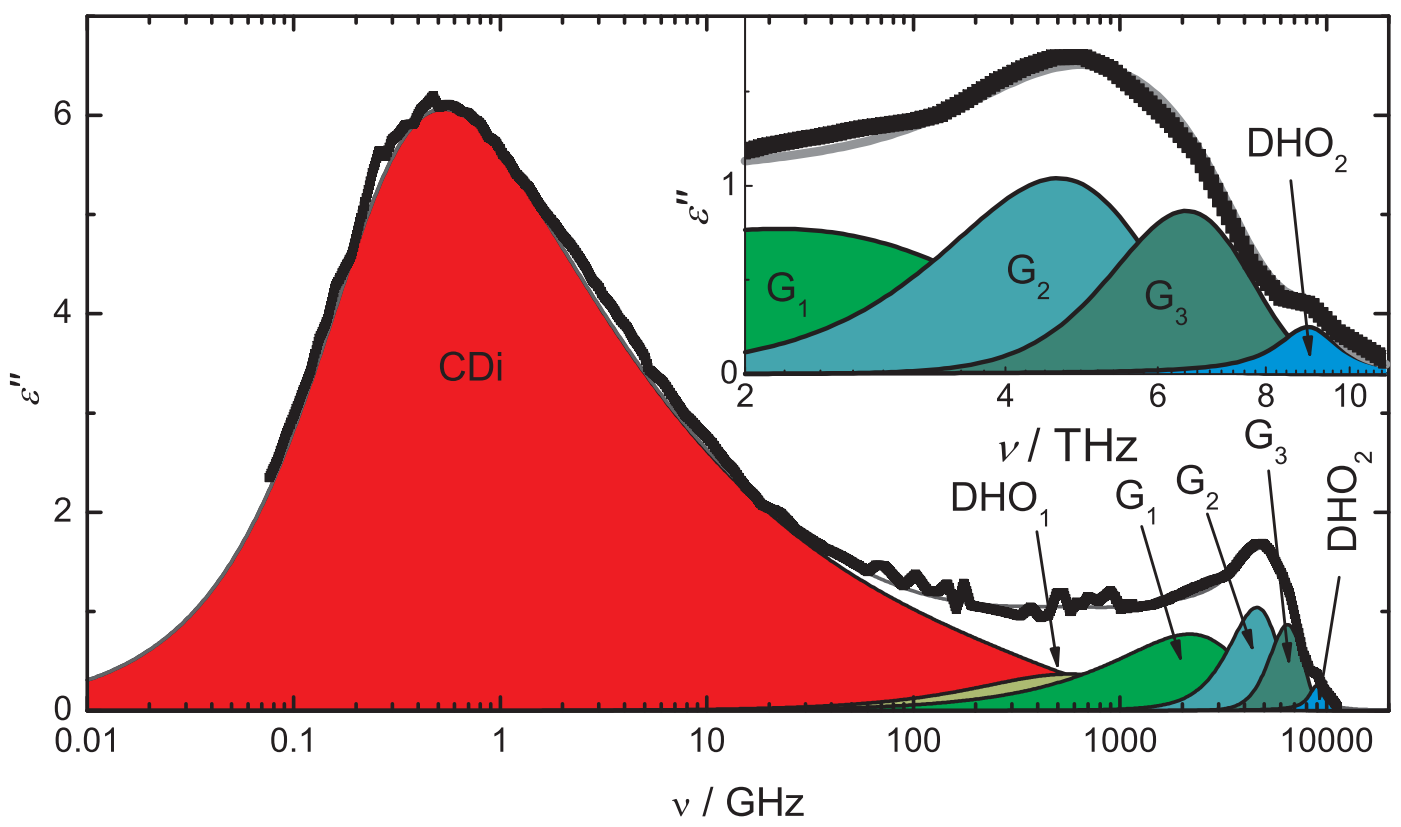

Figure 6: Dielectric loss spectrum of PAN at $25^{\circ} \mathrm{C}$. Black squares are resampled experimental data and the gray line represents the overall fit of the $\left(\mathrm{CDi}+\mathrm{DHO}_{1}+\mathrm{G}_{1}+\mathrm{G}_{2}+\mathrm{G}_{3}+\right.$ $\mathrm{DHO}_{2}$ ) model. Shaded areas indicate the contributions of the individual processes. Inset: expanded view of high frequency data.

\section{Discussion of "slow" dynamics ${ }^{1}$}

\subsection{Static Permittivity}

Before going on to discuss the details of the modes identified in the OKE and DR spectra at $\nu \lesssim 100 \mathrm{GHz}$ it is appropriate to consider the behavior of the latter in the low frequency limit. Dielectric spectroscopy is the only experimental method currently available for measuring the static permittivity (dielectric constant) of a conducting liquid. ${ }^{26}$ The values of the static permittivity $\left(\varepsilon_{\mathrm{s}}=\lim _{\nu \rightarrow 0} \varepsilon^{\prime}(\nu)=\sum_{j} S_{j}+\varepsilon_{\infty}\right)$ obtained for EAN and PAN from the fits of the present DR spectra are shown as a function of temperature in Fig. 7. As for other ILs, ${ }^{48}$ $\varepsilon_{\mathrm{S}}$ exhibits a monotonic (in fact approximately linear) decrease with increasing temperature, with $\mathrm{d} \varepsilon_{\mathrm{s}} / \mathrm{d} T=-0.13 \mathrm{~K}^{-1}(\mathrm{EAN})$ or $-0.09 \mathrm{~K}^{-1}(\mathrm{PAN})$. These large negative values of $\mathrm{d} \varepsilon_{\mathrm{s}} / \mathrm{d} T$ are about three times greater than for typical aprotic ILs ${ }^{48}$ but are similar to those of $\mathrm{H}-$ bonded molecular liquids such as alcohols, where they are ascribed to a partial break-up of

\footnotetext{
${ }^{1}$ Arbitrarily defined here as corresponding to $\nu \lesssim 100 \mathrm{GHz}$.
} 
the H-bond networks with increasing $T$. As would be expected from their protic character the values of $\varepsilon_{\mathrm{s}} \approx 27.3(\mathrm{EAN})$ and $23.4(\mathrm{PAN})$ at $25^{\circ} \mathrm{C}$ are considerably higher than those observed for aprotic ILs, which are typically $<20 .{ }^{48,52}$ It should be noted in passing that there is some uncertainty in the determination of $\varepsilon_{\mathrm{s}}$ for both EAN and PAN. This is because the dominant $\alpha$-relaxation (Section 4.2) slows down considerably (shifts to lower frequencies) at lower $T$, which means that $\varepsilon^{\prime}(\nu)$ does not reach its plateau within the accessible frequency range (Figs. 3a and 4a). Measurements at lower $\nu$ do not help because the dielectric signal is swamped by the conductivity contribution (eq 2). These difficulties, and the limited accuracy of existing dielectric instrumentation, readily account for the small differences (ca. 3-5\%) between the present values of $\varepsilon_{\mathrm{s}}$ and of $\mathrm{d} \varepsilon_{\mathrm{s}} / \mathrm{d} T$ for EAN and those of Weingärtner et al. ${ }^{15}$ (Fig. 7).

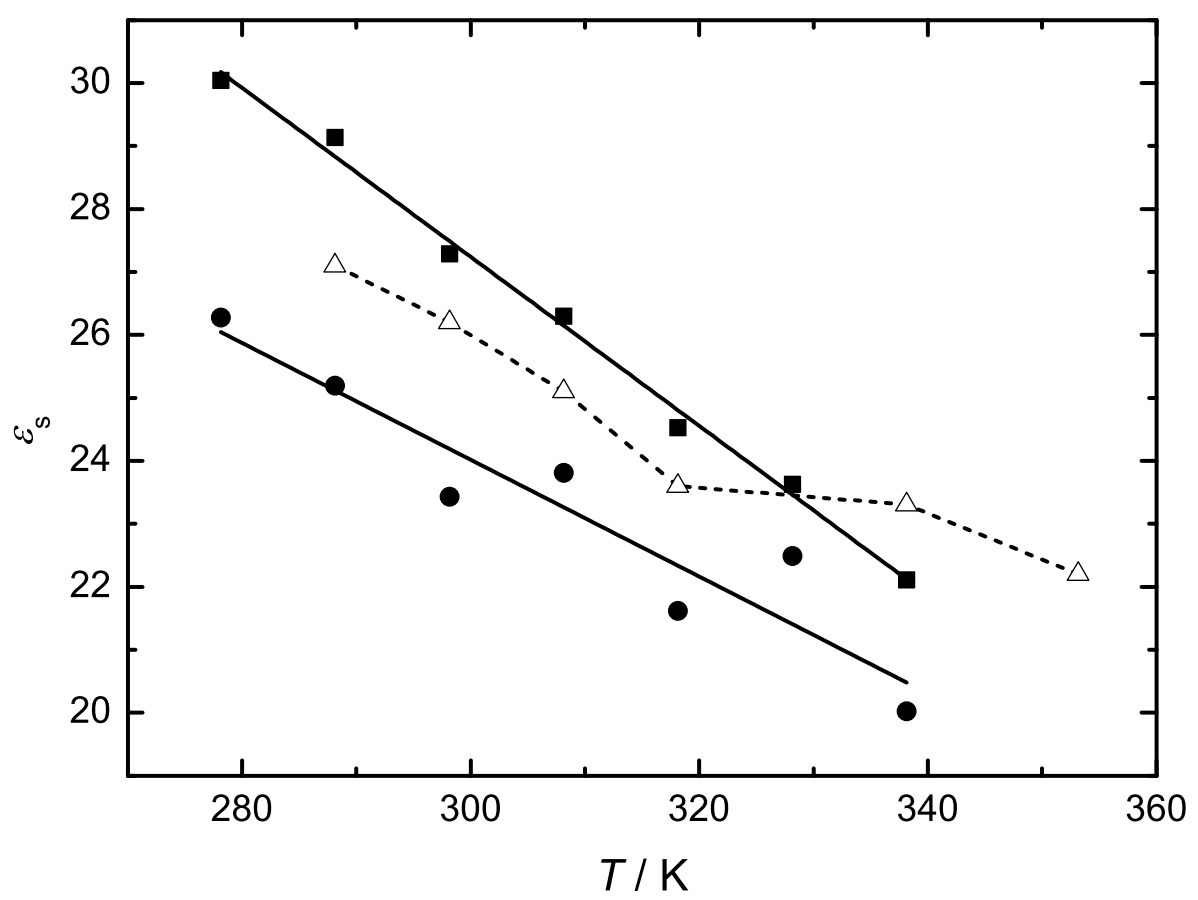

Figure 7: Present results for the static permittivity, $\varepsilon_{\mathrm{s}}$, of $\operatorname{EAN}(\mathbf{\square})$ and $\operatorname{PAN}(\mathbf{0})$, as a function of temperature; solid lines are linear regressions of the present data; open triangles $(\triangle)$ show the EAN values of Weingärtner et al. ${ }^{15}$ 


\subsection{The $\alpha$-Relaxation Process}

General Aspects. The dominant process in both the DR and OKE spectra of EAN and PAN in the low $\mathrm{GHz}$ region (Figs. 2 - 6) is the so-called $\alpha$-relaxation, ${ }^{51}$ which corresponds to the structural relaxation of (rotational motions of molecular-level species in) each liquid. ${ }^{53}$ Because of its three-fold symmetry axis, the nitrate anion has a zero dipole moment and thus will make no direct contribution to the DR spectra. On the other hand, due to its $\pi$-electrons $\mathrm{NO}_{3}^{-}$is highly polarizable and, because of its planar geometry, it has a high anisotropy $\left(\alpha_{\|} \approx\right.$ $\left.5.6 \AA^{3}, \alpha_{\perp} \approx 3.1 \AA^{3}\right) ;{ }^{54}$ the motions of $\mathrm{NO}_{3}^{-}$will therefore be a major contributor to the OKE spectra. Conversely, the ethyl- and propylammonium cations $\left(\mathrm{EtNH}_{3}^{+} \& \mathrm{PrNH}_{3}^{+}\right)$are only weakly polarizable and anisotropic but have considerable permanent dipole moments $(\mu \approx$ $3.9 \mathrm{D}$ and $6.8 \mathrm{D}$, respectively, from MOPAC calculations ${ }^{55}$ ), which make them significant contributors to the dielectric signal. In this sense, EAN and PAN can be viewed as almost perfect compounds for complementary investigation by OKE and DR spectroscopies. ${ }^{18}$

The amplitude of the $\alpha$-relaxation decreased considerably with increasing $T$ in both the OKE and DR spectra (Figs. 2 and 3). Furthermore, the peak maximum shifted from 0.8 to $6 \mathrm{GHz}$ in the OKE spectra (0.7 to $4 \mathrm{GHz}$ in the DR spectra) for EAN (Figs. 2 and 3) and from 0.2 to $2 \mathrm{GHz}$ in the DR spectra of PAN (Fig. 4). These features of the $\alpha$-relaxation are broadly similar to those observed for the corresponding process in imidazolium-based ILs. ${ }^{17}$ However, in contrast to the latter but as would be expected, there is no evidence in the present ILs of a sub- $\alpha$ process, which is thought to be a breathing mode of $\pi$-stacked cation aggregates. ${ }^{17}$

Relaxation Times. The relaxation times of the $\alpha$-process, $\tau_{\alpha}$, in the DR and OKE spectra of EAN and PAN decrease exponentially with increasing $T$ thus permitting application of the Arrhenius equation. As already noted (Section 3.1) the viscosities of both liquids follow the VFT relation over the same temperature range. As a consequence, Stokes-Einstein-Debye $(\mathrm{SED})$ plots of $\tau_{\alpha}^{\mathrm{DR}}(\mathrm{EAN}), \tau_{\alpha}^{\mathrm{OKE}}(\mathrm{EAN})$ and $\tau_{\alpha}^{\mathrm{DR}}(\mathrm{PAN})$ against $\eta / k_{\mathrm{B}} T$ depart from linearity 
at low $T,{ }^{18}$ (for convenience these plots are reproduced in Fig. S2) which implies a decoupling of the molecular-level rotational motions from the bulk viscosity. At higher $T$ the effective volumes of rotation, $V_{\text {eff }}$, extracted from the slopes of the SED plots were rather small and were not compatible with the probable rotational motions of the anions (out-of-plane rotation for $\mathrm{NO}_{3}^{-}$) or the cations (end-over-end rotation for $\mathrm{RNH}_{3}^{+}$). ${ }^{18}$ The non-linearity of the SED plots is instead consistent with a high degree of rotational cooperativity between the anions and cations, rather than the simple independent rotational diffusion assumed in the SED theory.

For EAN, further evidence for such cooperative motions comes from a comparison of DRS with time-resolved femtosecond-IR spectroscopy (fs-IRS). ${ }^{56}$ As fs-IRS probes the rotation of the $\mathrm{N}-\mathrm{H}$ bonds, it is also sensitive to the rotations of the $\mathrm{EtNH}_{3}^{+}$cation, which manifests itself in an activation energy $\left(E_{\mathrm{A}}^{\mathrm{IR}}=21.4 \pm 0.6 \mathrm{~kJ} \mathrm{~mol}^{-1}\right)^{56}$ that is virtually identical with the present results $\left(E_{\mathrm{A}}^{\mathrm{DR}}=21.6 \pm 0.3 \mathrm{~kJ} \mathrm{~mol}^{-1}\right)$. Within the rotational diffusion model, where specific interactions of the rotating entity with its surroundings are ignored, a ratio of $\tau_{\alpha, \max }^{\mathrm{DR}} / \tau^{\mathrm{IR}}=3$ is expected as the fs-IR relaxation times, $\tau^{\mathrm{IR}}$, are of second rank in contrast to the first-rank relaxation time, $\tau_{\alpha}^{\mathrm{DR}}$, measured via DRS. ${ }^{25}$ For EAN, however, the observed ratio has an average value of $1.4,{ }^{57}$ which is consistent with the $\alpha$-relaxation process occurring via a jump mechanism, as previously proposed for aprotic ILs, ${ }^{17,58}$ rather than through rotational diffusion. In water, where molecular reorientation is also thought to take place via large-angle jumps, ${ }^{59}$ the ratio of first- to second-rank rotational correlation times is $\sim 2$ from which it was concluded that the relaxation rate in water is determined by the rate of H-bond breaking. ${ }^{60}$ It seems likely that this is also the case in EAN, with the even lower value of $\tau_{\alpha, \max }^{\mathrm{DR}} / \tau^{\mathrm{IR}}$ reflecting the very strong and multiple cation-anion $\mathrm{H}$ bond interactions. Knowledge of the ratio $\tau_{\alpha, \max }^{\mathrm{DR}} / \tau^{\mathrm{IR}}$ permits ${ }^{56}$ calculation of the jump angle, yielding a result of $106^{\circ}$. This value is almost identical with the tetrahedral angle of the ammonium group, which is again consistent with strong, directed H-bond interactions between $\mathrm{EtNH}_{3}^{+}$and $\mathrm{NO}_{3}^{-}$. 
This conclusion is also supported by the similarity of the activation energies for both EAN and PAN obtained from the temperature dependence of $\tau_{\alpha}^{\mathrm{DR}}\left(E_{\mathrm{A}}^{\mathrm{DR}} / \mathrm{kJ} \mathrm{mol}^{-1}=21.6 \pm 0.3\right.$ $(\mathrm{EAN})$ or $26.0 \pm 1.7(\mathrm{PAN}))$ and $\tau_{\alpha}^{\mathrm{OKE}}\left(E_{\mathrm{AKE}}^{\mathrm{OKJ}} / \mathrm{kJ} \mathrm{mol}^{-1}=24.0(\mathrm{EAN})\right.$ or $\left.25.5(\mathrm{PAN})\right) .{ }^{18,20}$ These relatively large $E_{\mathrm{A}}$ values, and particularly their similarity, indicate a high degree of cooperativity between the rotating species and their neighbors. Such cooperativity is also implied by the similarity of $\tau_{\alpha}^{\mathrm{DR}}$ and $\tau_{\alpha}^{\mathrm{OKE}}$ at all $T$, which suggests that anion dynamics are slowed down to the time scale of cation reorientation as a result of strong inter-ionic interactions.

Amplitudes. The amplitudes (intensities) of the $\alpha$-relaxation in the DR spectra, $S_{\alpha}^{\mathrm{DR}}$, arise from the motions of the dipolar species present. In a PIL these include cooperative fluctuations of the H-bond network, including H-bond making and breaking. For EAN and PAN, $S_{\alpha}^{\mathrm{DR}}$ is dominated by the rotational motions of $\mathrm{EtNH}_{3}^{+}$and $\mathrm{PrNH}_{3}^{+}$, which have gas phase dipole moments, $\mu=3.86 \mathrm{D}^{61}$ and $6.84 \mathrm{D}\left(\mathrm{MOPAC}^{55}\right)$, respectively. The $\mathrm{NO}_{3}^{-}$ion has no dipole moment and thus makes no direct contribution to the DR spectrum. However, the translational motions of the ions may contribute in the same frequency range, ${ }^{44}$ as well as the rotational/translational motions of any ion pairs (IPs) or larger clusters that have $\mu \neq 0$ and an appropriate lifetime. The corresponding OKE amplitude, $S_{\alpha}^{\mathrm{OKE}}$, arises from changes in polarizability anisotropy, which — as argued above in this Section-mainly reflects the motions of the $\mathrm{NO}_{3}^{-}$ion, along with any IPs or larger clusters containing $\mathrm{NO}_{3}^{-}$that have the relevant characteristics. Note that, as with all neat ILs investigated to date, ${ }^{48}$ there is no evidence in the DR spectra for the existence of discreet IPs in the present PILs; furthermore, any larger clusters (if present) detected by DR and OKE will not necessarily be identical. Nor is it known a priori what the relative contributions to $S_{\alpha}$ might be due to the ions, IPs and clusters (if present). 
Quantitative evaluation of $S_{\alpha}^{\mathrm{DR}}$ was made via the equation

$$
\frac{\varepsilon_{\mathrm{s}}+A_{j}\left(1-\varepsilon_{\mathrm{s}}\right)}{\varepsilon_{\mathrm{s}}} \cdot S_{j}=\frac{N_{\mathrm{A}} c_{j}}{3 k_{\mathrm{B}} T \varepsilon_{0}} \cdot \mu_{\mathrm{eff}, j}^{2}
$$

which relates the amplitude of a relaxation process, $S_{j}$, to the effective dipole moment, $\mu_{\mathrm{eff}, j}$, of the relaxing species $j$ with concentration $c_{j} .{ }^{62}$ In eq $13 N_{\mathrm{A}}$ and $k_{\mathrm{B}}$ are the Avogadro and Boltzmann constants, respectively. To apply eq 13 the shapes of $\mathrm{EtNH}_{3}^{+}$and $\mathrm{PrNH}_{3}^{+}$were approximated as prolate ellipsoids using the equations for the geometry factor, $A_{j}$, given by Scholte. ${ }^{63}$ The values of $\mu_{\text {eff }}$ so obtained decrease linearly with increasing $T$ (Fig. 8).

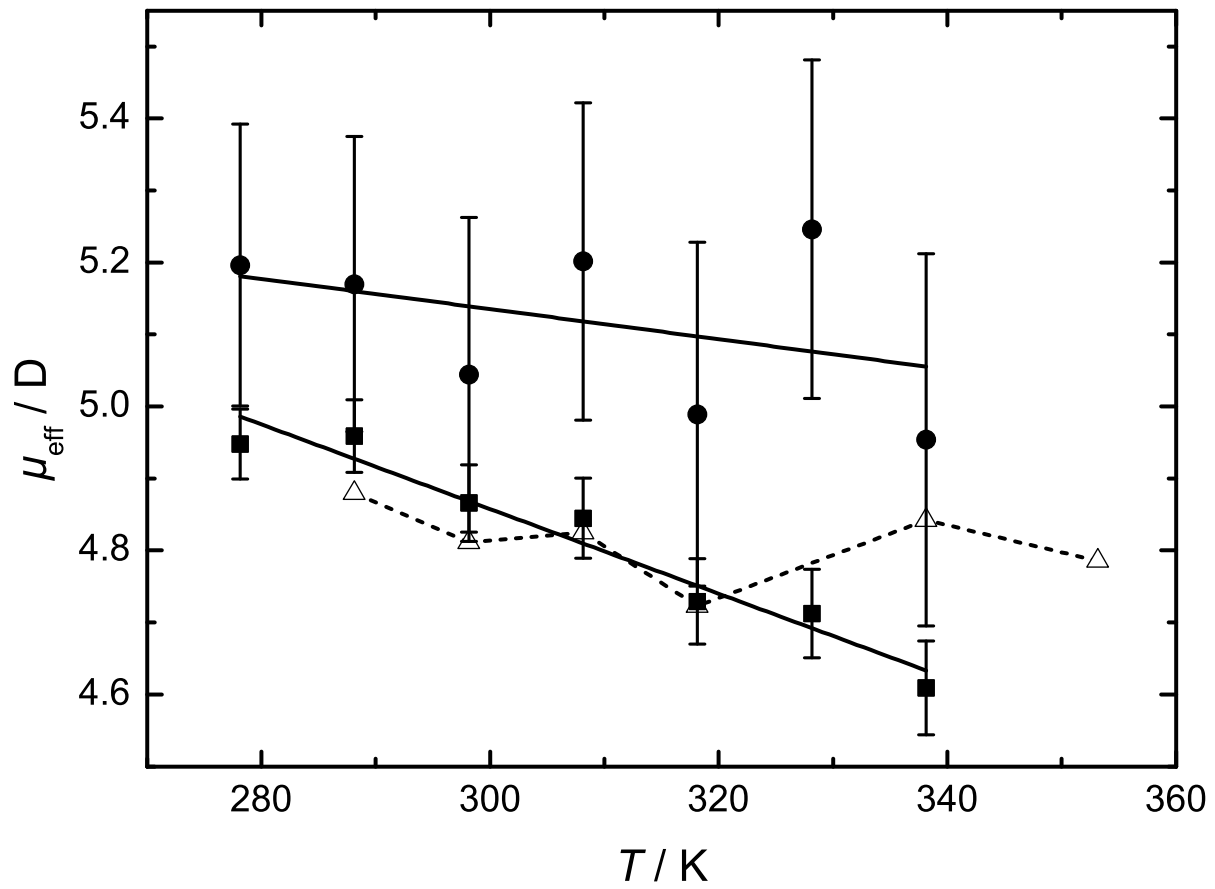

Figure 8: Present results for the effective dipole moment, $\mu_{\mathrm{eff}}$, of the $\alpha$ process as a function of temperature: EAN ( $\mathbf{\square})$ and PAN $(\mathbf{O})$. Solid lines represent linear fits of the present data; open triangles $(\triangle)$ are literature values for $\operatorname{EAN}(\triangle) .{ }^{15}$

Within the likely experimental errors, up to $45^{\circ} \mathrm{C}$ the present $\mu_{\text {eff }}$ values agree well with those of Weingärtner et al. ${ }^{15}$ although the latter's values at 65 and $80{ }^{\circ} \mathrm{C}$ differ significantly from the trend of the present results. For EAN, $\mu_{\text {eff }}$ coincides with the apparent dipole moment, $\mu_{\mathrm{app}}=4.9 \mathrm{D}$, of $\mathrm{EtNH}_{3}^{+}$calculated by MOPAC, ${ }^{55}$ consistent with the assumption that $S_{\alpha}^{\mathrm{DR}}$ mostly arises from cation reorientation. The value of $\mu_{\text {eff }}$ for PAN is signifi- 
cantly higher than that for EAN (Fig. 8) consistent with its higher MOPAC-calculated value $\left(\mu_{\mathrm{app}}=7.9 \mathrm{D}\right) .{ }^{64}$ The decrease of $\mu_{\mathrm{eff}}$ with increasing $T$ contrasts with all aprotic imidazolium-based ILs studied to date, for which $\mu_{\text {eff }}$ was found to be independent of $T,{ }^{48}$ which has been interpreted as indicating an absence of structural change. The negative values of $\mathrm{d} \mu_{\text {eff }} / \mathrm{d} T$ of EAN and possibly PAN (Fig. 8) therefore imply increasing dipole-dipole interactions in these PILs at lower $T$. This is consistent with the formation of higher order clusters in these ILs and perhaps also with increasing micro-heterogeneity as proposed by Hayes et al. ${ }^{9}$ Whatever the exact nature of the orientational correlations of the dipoles, it is likely that they are also responsible for the decoupling of viscosity and rotational motions inferred from the relaxation time. The fact that $\varepsilon_{\mathrm{S}}(\mathrm{PAN})<\varepsilon_{\mathrm{S}}(\mathrm{EAN})$ despite having a larger value of $\mu_{\text {eff }}$ can be reasonably ascribed to the lower dipole density in PAN.

\section{Discussion of "fast" dynamics ${ }^{2}$}

\subsection{EAN}

The observed intensity of the broadband DR and OKE spectra of ILs in the frequency range $0.1<\nu / \mathrm{THz}<13$ (ca. 3 to $400 \mathrm{~cm}^{-1}$ ) mostly arises from librations and intermolecular vibrations. ${ }^{17,65,66}$ For PILs, low energy intramolecular vibrations and proton transfers may also contribute. Because of the strong inter-ionic interactions in PILs and the probably extensive coupling and overlapping of rotational, vibrational and translational modes, ${ }^{39}$ unambiguous decomposition and assignment of modes in this region is especially difficult (Section 3.2). Nevertheless, the different "selection rules" for OKE, sensitive to fluctuations of the polarizability anisotropy, and DRS, probing dipole fluctuations, means that a combination of OKE and DR spectroscopies can provide valuable insights into the dynamics of PILs.

Based on DFT calculations of small PIL clusters, Fumino et al. ${ }^{13}$ assigned contributions in the 50 to $400 \mathrm{~cm}^{-1}$ range of their FIR absorption spectra of EAN and related compounds

\footnotetext{
${ }^{2}$ Arbitrarily defined here as corresponding to $\nu \gtrsim 100 \mathrm{GHz}$.
} 
to intermolecular H-bond bending and stretching (Table 1), drawing particular attention to the similarity of these modes to those observed for water. ${ }^{66}$ However, one may argue whether symmetric and asymmetric H-bond stretching modes can be distinguished in the spectra of H-bonding liquids due to the generally fast dynamic exchange of different H-bonded configurations. ${ }^{67}$ Even more, in addition to such vibrations, intensity in this region must also reflect the librational motions of $\mathrm{NO}_{3}^{-}$and $\mathrm{EtNH}_{3}^{+}$. The contributions of these motions to the OKE and DR spectra can be estimated using the free-rotator approximation of Bartoli. ${ }^{68}$ This model predicts that because of their moments of inertia $\left(\mathrm{EtNH}_{3}^{+}: I_{B}=100.6 \cdot 10^{-47} \mathrm{~kg} \mathrm{~m}{ }^{2}\right.$, $\left.I_{C}=114.4 \cdot 10^{-47} \mathrm{~kg} \mathrm{~m}^{2} ; \mathrm{NO}_{3}^{-}: I_{A}=I_{B}=61.9 \cdot 10^{-47} \mathrm{~kg} \mathrm{~m}^{2}\right)^{55}$ the libration frequencies of $\mathrm{NO}_{3}^{-}$and $\mathrm{EtNH}_{3}^{+}$should be reduced by factors of $\sim 7$ and $\sim 9$, respectively, compared to water. Using the value of $\sim 680 \mathrm{~cm}^{-1}$ for the libration mode of water ${ }^{69}$ and assuming for simplicity that the same mean-square torque is acting, values of $\sim 2.9 \mathrm{THz}\left(97 \mathrm{~cm}^{-1}\right)$ for $\mathrm{NO}_{3}^{-}$and $\sim 2.3 \mathrm{THz}\left(76 \mathrm{~cm}^{-1}\right)$ for $\mathrm{EtNH}_{3}^{+}$are obtained. It is therefore essential to consider possible librational contributions to the FIR spectra. A full discussion of the present assignment of the observed modes in the OKE and DR spectra is given below. We reiterate here, see Section 3.2., that the present approach creates a somewhat simplified picture of PIL dynamics as in reality all motions are strongly coupled. But as long as computer simulations or theoretical approaches do not yield quantitative agreement an evaluation of experimental spectra along the latter route is equally problematic.

OKE Fits. As discussed above, the high-frequency portion of the OKE spectra for EAN was best fitted by a model consisting of a damped harmonic oscillator, $\mathrm{DHO}_{1}($ at $\sim 1.5 \mathrm{THz}$ ), two Gaussians, $\mathrm{G}_{1}(\sim 2 \mathrm{THz})$ and $\mathrm{G}_{2}^{\prime}(\sim 5 \mathrm{THz})$, plus an additional mode $\left(\mathrm{DHO}_{2} ; 7.9 \mathrm{THz}\right)$ that accounted for the small peak in the OKE spectra (Fig. 5a, inset), which also shows up in the DR spectra as a shoulder (Fig. 5b, inset) and which is associated with ethyl-group torsion (Table 1). ${ }^{13}$

Figure 9 displays the temperature dependence of the amplitudes, bandwidths and reso- 
Table 1: Comparison for EAN of the resonance frequencies, $\bar{\nu}_{0} / \mathbf{c m}^{-1}$, of the present $\mathrm{OKE}$ and $\mathrm{DR}$ modes, $\mathrm{DHO}_{1}, \mathrm{G}_{1}, \mathrm{G}_{2}, \mathrm{G}_{2}^{\prime}, \mathrm{G}_{3}$ (intermolecular) and $\mathrm{DHO}_{2}$ (intramolecular) at $65^{\circ} \mathrm{C}$ with the FIR absorption modes reported at $80^{\circ} \mathrm{C}$ by Fumino et al. ${ }^{13}$

\begin{tabular}{|c|c|c|c|c|c|}
\hline \multirow[t]{2}{*}{ Mode } & \multirow[t]{2}{*}{$\bar{\nu}_{0}^{\mathrm{OKE}}$} & \multirow[t]{2}{*}{$\bar{\nu}_{0}^{\mathrm{DR}}$} & \multirow[t]{2}{*}{$\bar{\nu}_{0}^{\mathrm{FIR} 13}$} & \multicolumn{2}{|c|}{ Assignment } \\
\hline & & & & Fumino et al. ${ }^{13 a}$ & This work \\
\hline $\mathrm{DHO}_{1}$ & 49.4 & $49.4^{b}$ & $-^{c}$ & $-^{c}$ & H-bond bending \\
\hline $\mathrm{G}_{1}$ & 68.4 & $68.4^{b}$ & 60 & H-bond bending & $\mathrm{NO}_{3}^{-}$librations \\
\hline $\mathrm{G}_{2}$ & - & 149.1 & 132 & $\begin{array}{l}\text { Symmetric H-bond } \\
\text { stretching }\end{array}$ & H-bond stretching \\
\hline $\mathrm{G}_{2}^{\prime}$ & 166.1 & - & $-^{c}$ & $--^{c}$ & $\begin{array}{l}\text { Unresolved combination } \\
\text { of } \mathrm{G}_{2} \& \mathrm{G}_{3} \text {, see text }\end{array}$ \\
\hline $\mathrm{G}_{3}$ & - & 184.8 & 199 & $\begin{array}{l}\text { Asymmetric H-bond } \\
\text { stretching }\end{array}$ & $\mathrm{RNH}_{3}^{+}$librations \\
\hline $\mathrm{DHO}_{2}$ & 263.3 & 263.3 & 265.2 & Ethyl group torsion & Ethyl group torsion \\
\hline
\end{tabular}

nance frequencies of the proposed high frequency processes. The OKE amplitudes (Fig. 9d) are only weakly affected by $T: S_{\mathrm{G} 1}(\boldsymbol{\Lambda})$ and $S_{\mathrm{G} 2^{\prime}}(\boldsymbol{\vee})$ increase slightly, while $S_{\mathrm{DHO} 1}(\square)$ is more or less constant over the investigated range. On the other hand, the damping constants (Fig. 9e) differ in their temperature dependence: $\gamma_{\mathrm{G} 1}(\boldsymbol{\Delta})$ and $\gamma_{\mathrm{G} 2^{\prime}}(\boldsymbol{\vee})$ increase with increasing $T$ (with a larger slope for the latter), whereas $\gamma_{\mathrm{DHO} 1}(\square)$ is approximately constant. The OKE resonance frequencies (Fig. 9f) of all processes decrease with increasing $T$. For librations this decrease in $\nu_{0, j}$ with increasing $T$ is generally explained by the general increase in molecular motions, and the weakening of the surrounding cage as the density decreases. However, these same factors also lower the force constants of intermolecular vibrations, such as H-bond bending or stretching, which in turn lowers $\nu_{0}$ as $T$ increases. It is therefore not possible to use these observations to determine whether these modes $\left(\mathrm{G}_{1}, \mathrm{G}_{2}^{\prime}, \mathrm{DHO}_{1} \& \mathrm{DHO}_{2}\right)$ are librations or vibrations.

The location of the resonance frequency (Table 1$)$ of the $G_{1}$ mode in OKE $\left(68.4 \mathrm{~cm}^{-1}\right.$ at $338 \mathrm{~K}$ ) is compatible with the FIR mode at $\sim 60 \mathrm{~cm}^{-1}$ at $353 \mathrm{~K}$, assigned by Fumino et al. ${ }^{13}$ to H-bond bending. However, the present OKE measurements indicate that this mode 
mainly arises from librational motions of the nitrate anion as the polarizability of this ion is highly anisotropic but not that of the cation. The justifications for this assignment are as follows. First, OKE is primarily sensitive to rotational motions and thus would not be expected to detect $H$-bond bending. ${ }^{70}$ Second, the OKE spectra of all liquids show a pronounced librational peak in this frequency range, independent of the presence or absence of H-bonds. ${ }^{65,71}$ Finally, the small increase in $S_{\mathrm{G} 1}$ with increasing $T$ is expected for librational motions, due to increasing thermal motions, whereas for vibrations, amplitudes usually decrease. Although for pyrrolidinium, tetraalkylammonium and tetraalkylphosphonium ILs the mode at $\sim 60 \mathrm{~cm}^{-1}$ could be associated with interionic stretching vibrations ${ }^{72,73}$ this unlikely for EAN (and PAN) as here the masses of the involved ions are too small for such a low frequency.

With regard to the $\mathrm{G}_{2}^{\prime}$ mode in the OKE spectra it can be speculated that it is related to cation motions as $S_{\mathrm{G} 2^{\prime}} \ll S_{\mathrm{G} 1}$ (Fig. 5a, inset), in line with the respective polarizability anisotropies of $\mathrm{NO}_{3}^{-}$and $\mathrm{EtNH}_{3}^{+}$(Section 4.2). The frequency maximum of $\mathrm{G}_{2}^{\prime}$ in the OKE spectra lies between those of the $\mathrm{G}_{2}$ and $\mathrm{G}_{3}$ modes in the DR spectra. This suggests that $\mathrm{G}_{2}^{\prime}$ is a composite mode that can be resolved in the DR but not in the OKE spectra. Furthermore, the slight increase in $S_{\mathrm{G}^{\prime}}$ with increasing $T$ (Fig. 9d) implies that the librational portion (as discussed later) predominates in $\mathrm{G}_{2}^{\prime}$, consistent with the fact that OKE is mainly sensitive to rotational motions.

DRS Fits. As described in Section 3.2 the high-frequency part of the DR spectra of EAN was fitted by a model consisting of a damped harmonic oscillator, $\mathrm{DHO}_{1}(\sim 1.5 \mathrm{THz})$, three Gaussians, $\mathrm{G}_{1}(\sim 2.0 \mathrm{THz}), \mathrm{G}_{2}(\sim 4.5 \mathrm{THz})$, and $\mathrm{G}_{3}(\sim 5.6 \mathrm{THz})$ plus an additional mode, $\mathrm{DHO}_{2}(7.9 \mathrm{THz})$, that is associated with the intramolecular torsion of the ethyl group of $\mathrm{EtNH}_{3}^{+}$(Table 1). ${ }^{13}$

It is reiterated here (see also Section 3.2) that while this model provided the best fit of the observed DR spectra is not the only possible model. This uncertainty is due in part to 

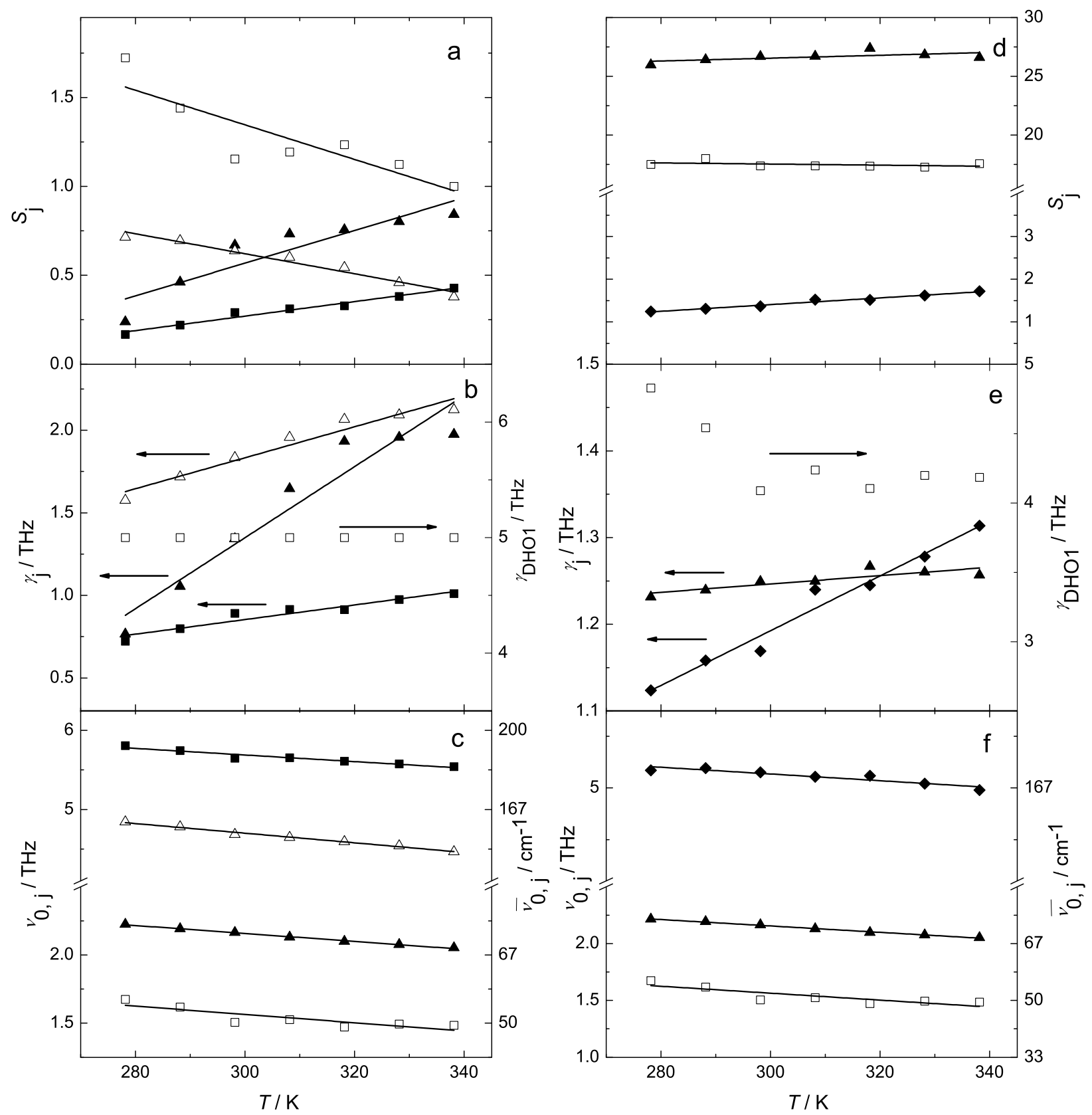

Figure 9: Fit parameters for the high-frequency resonance processes of EAN as a function of temperature: (a) amplitudes, $S_{j}$, (b) damping constants, $\gamma_{j}$, and (c) resonance frequencies, $\nu_{0, j}$, of the $\mathrm{DHO}_{1}(\square), \mathrm{G}_{1}(\mathbf{\Delta}), \mathrm{G}_{2}(\triangle)$ and $\mathrm{G}_{3}(\mathbf{\square})$ modes in the DR spectra; (d) $S_{j},(\mathbf{e}) \gamma_{j}$, and $(\mathbf{f}) \nu_{0, j}$, of the $\mathrm{DHO}_{1}(\square), \mathrm{G}_{1}(\boldsymbol{\Delta}), \mathrm{G}_{2}^{\prime}(\boldsymbol{\vee})$ modes in the OKE spectra. Solid lines are linear regressions of the data.

the strongly overlapping contributions from coupled multiple-particle interactions and also because of significant technical limitations on the accuracy of the spectra particularly in the low- $\mathrm{THz}$ range. These problems evidence themselves by a small underestimation of the 
observed intensities at intermediate frequencies: $50 \lesssim \nu / \mathrm{GHz} \lesssim 1000$ (Fig. 5). Nevertheless, as argued in Section 3.2, inclusion of further processes, e.g. a constant loss term ${ }^{18}$ or an additional Debye function at $\sim 80 \mathrm{GHz}$, yielded no improvement in the fits. Fortunately, the imperfectly-fitted intermediate frequency range is sufficiently well separated from the high frequency $(\nu>1 \mathrm{THz})$ region, that is of special interest here because of what it may reveal about the similarities (or otherwise) between EAN and water. ${ }^{13,66,69}$

All the fit parameters for the modes in this region of the dielectric spectra of EAN (Figs. 9a,b,c) and PAN (Fig. 12 \& Table S5) show reasonably smooth trends with temperature. Thus, the resonance frequencies decrease slightly with increasing $T$ for all modes (Figs. 9c \& 12b), as for the OKE fits (Fig. 9f). But again this trend does not distinguish between librational and vibrational contributions because, as observed for water, ${ }^{74,75} \nu_{0}$ of H-bond stretching also decreases with increasing $T$. On the other hand, the corresponding DR amplitudes (Fig. 9a) differ in their temperature dependence: $S_{\mathrm{G} 1}(\boldsymbol{\Delta})$ and $S_{\mathrm{G} 3}(\boldsymbol{\square})$ increase with increasing $T$, whereas $S_{\mathrm{DHO} 1}(\square)$ and $S_{\mathrm{G} 2}(\triangle)$ decrease. This must mean, that in contrast with the model of Fumino et al. ${ }^{13}$ not all of the Gaussian modes can be due to intermolecular H-bond vibrations. If so, the present Gaussian modes would be expected to decrease with increasing $T$ due to the weakening of the H-bonds.

Whilst $\mathrm{G}_{1}$ in OKE (Fig. 5a) can be confidently assigned to nitrate libration, its relatively weak equivalent in DRS (Fig. 5b) may be related to the collision-induced part of this mode. The reasons for this tentative assignment are: first because $\nu_{\mathrm{G} 1}^{\mathrm{DR}} \approx \nu_{\mathrm{G} 1}^{\mathrm{OKE}}$ and secondly because $S_{\mathrm{G} 1}^{\mathrm{DR}}$ increases with $T$ (Fig. 9a, $\left.\mathbf{\Delta}\right)$. The latter is what would be expected for an interaction-induced contribution (and a libration) because of the increasing collision rate due to increasing thermal motions. For H-bond vibrations the opposite trend is expected because of the decreasing liquid density and H-bond weakening. A similar situation is found for $\mathrm{G}_{3}$ : because its amplitude increases with increasing $T$ (Fig. 9a, $\mathbf{\square}$ ), it is reasonable to associate it with cation librations. ${ }^{76}$

In contrast to $S_{\mathrm{G} 1}$ and $S_{\mathrm{G} 3}$, the magnitude of $S_{\mathrm{G} 2}$ decreases with increasing $T$ (Fig. 9a, 
$\triangle)$. Such a trend is compatible with H-bond vibrations, as observed for water. ${ }^{13,76}$ If so, $\mathrm{G}_{2}$ can be attributed to H-bond stretching, which is more energetic than H-bond bending. This means H-bond bending (if such a contribution exists) might be a possible origin of the $\mathrm{DHO}_{1}$ mode. This assignment of $\mathrm{DHO}_{1}$ is consistent with that of Fumino et al. ${ }^{13}$ and is supported by the drop in $S_{\mathrm{DHO}_{1}}$ with increasing $T$ (Fig. $9 \mathrm{a}, \square$ ). It should be noted, however, that $\mathrm{DHO}_{1}$ may also include a contribution from a possible CL mode.
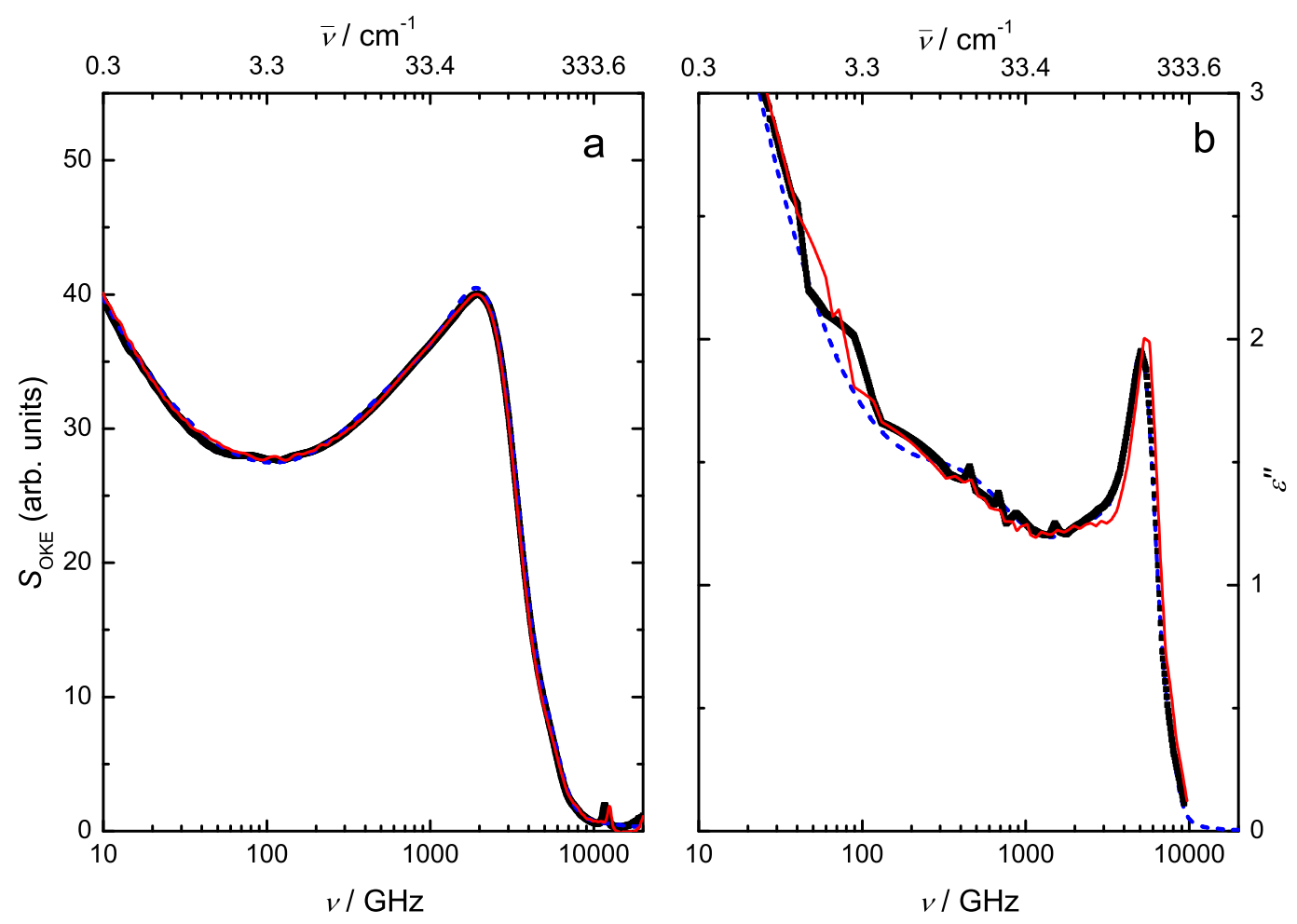

Figure 10: (a) OKE and (b) DR spectra of EAN (red solid lines) and $\mathrm{d}_{3}$-EAN (ם) with the corresponding total fit of $\mathrm{d}_{3}$-EAN (dashed blue lines) at $25^{\circ} \mathrm{C}$.

\subsection{Deuterated EAN}

The $\mathrm{H}$ atoms of the ammonium group of EAN were $\sim 91 \%$ replaced by deuterium atoms, corresponding to an average composition of $\left[\mathrm{EtND}_{2.7} \mathrm{H}_{0.3}\right]^{+}$which, for convenience, will referred to throughout as $\mathrm{d}_{3}$-EAN. Due to time and material constraints, DR and OKE spectra of $\mathrm{d}_{3}$-EAN were recorded only at $25^{\circ} \mathrm{C}$. Both spectra were found to be fitted satisfactorily with the same models used for EAN (Section 3.2). 
OKE Spectrum. The OKE spectrum of $\mathrm{d}_{3}$-EAN is compared to that of EAN in Fig. 10a. The two $25^{\circ} \mathrm{C}$ spectra match almost perfectly, showing no isotopic shift (IS) and providing nearly identical fit parameters (Table S3). Although the calculated IS for a H-bond vibration $(\sim 2 \%$, see below) is at the present detection limit, the close similarity of the OKE spectra of EAN and $d_{3}$-EAN (Fig. 10a) is compatible with the assignment of $\mathrm{G}_{1}$ (the dominant mode at $\sim 68 \mathrm{~cm}^{-1}, 2 \mathrm{THz}$, Fig. 5a) to nitrate libration. However, it must be noted that almost no ISs are observed for H-bond vibrations in water ${ }^{69}$ so the absence of a detectable IS for $\mathrm{G}_{1}\left(\mathrm{EAN}, \mathrm{d}_{3}-\mathrm{EAN}\right)$ is not definitive.

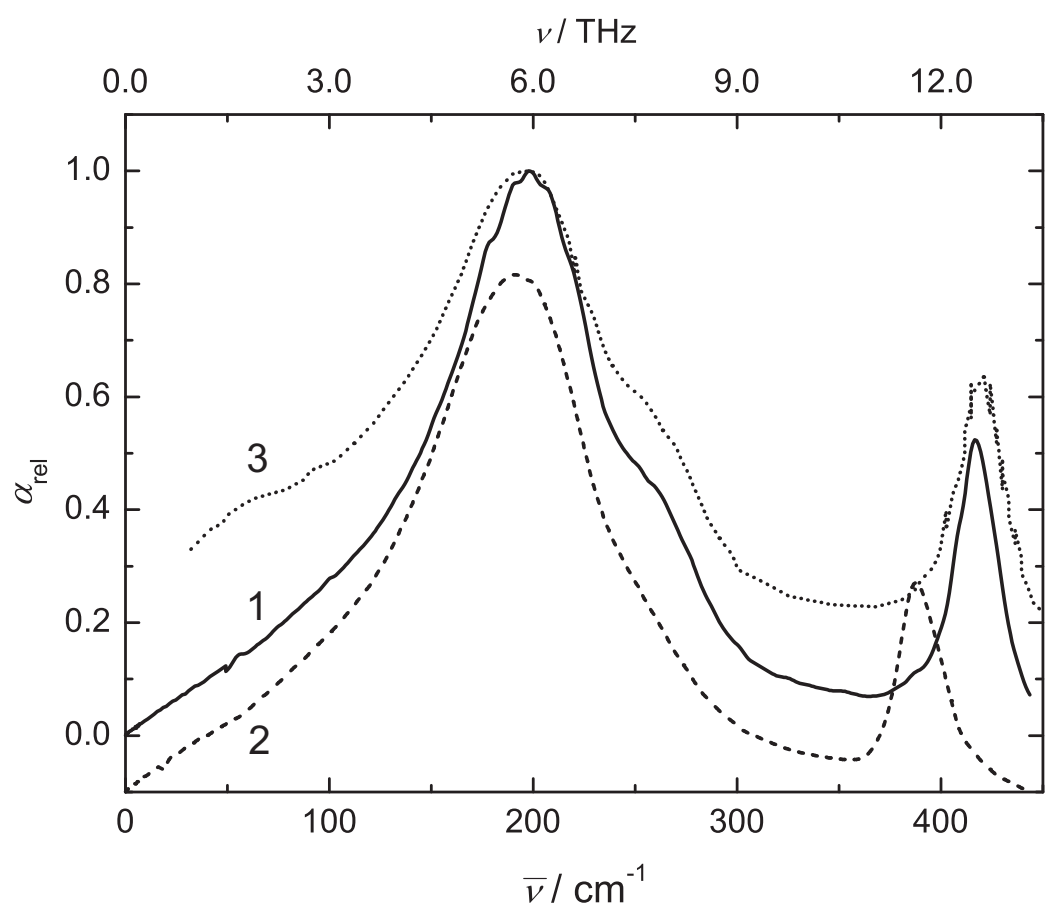

Figure 11: FIR absorption spectra, as $\alpha_{\text {rel }}(\bar{\nu})$, of EAN (1, solid line) and $\mathrm{d}_{3}$-EAN (2, dashed line) at $25^{\circ} \mathrm{C}$ (this work) and of EAN at $80{ }^{\circ} \mathrm{C}\left(3\right.$, dotted line). ${ }^{13}$ For visual clarity a negative offset has been applied to the spectrum of $\mathrm{d}_{3}$-EAN.

DR Spectrum. The situation is different for the dielectric spectrum. Figure 11 compares the present $\hat{\varepsilon}(\nu)$ data for $\mathrm{d}_{3}$-EAN and EAN at $25^{\circ} \mathrm{C}$ with the FIR results for EAN at $80{ }^{\circ} \mathrm{C}$ obtained by Fumino et al. ${ }^{13}$ For comparison purposes the present spectra have been converted to the relative absorption format, $\alpha_{\text {rel }}(\bar{\nu})$, after subtraction of $S_{\alpha}$ from the total intensity. Both of the EAN spectra are in qualitative agreement, but even when allowing 
for a possible contribution of $S_{\alpha}$ to the spectrum of Fumino et al. ${ }^{13}$ (curve 3 of Fig. 11) it appears that the intensity observed by them is considerably overestimated at $\bar{\nu}<100 \mathrm{~cm}^{-1}$. This is not a criticism of the work of Fumino et al. but rather a reflection of the difficulties of measurements in this frequency range, which has implications for the fitting of such spectra.

Comparison of curves $1 \& 2$ of Fig. 11 provides information on the EAN/d $\mathrm{d}_{3}$-EAN isotope shifts: A strong IS is observed for the pronounced resonance at $\sim 420 \mathrm{~cm}^{-1}$, associated with the intramolecular bending motion of the cation, ${ }^{13}$ which is shifted to $\sim 380 \mathrm{~cm}^{-1}$ by deuteration. A smaller but still significant IS is also observed for the peak of $\alpha_{\text {rel }}(\bar{\nu})$ at $\sim 200 \mathrm{~cm}^{-1}$, which corresponds to the peak in $\varepsilon^{\prime \prime}(\nu)$ at $\sim 5.5 \mathrm{THz}$ (Fig. 10b). If $\mathrm{G}_{2}$ is due to H-bond stretching and $\mathrm{G}_{3}$ to cation librations (as proposed in Section 5.1) the effects of deuteration on these two modes should differ from each other. For H-bond stretching an IS of $\left(\mu_{\mathrm{EAN}}^{\prime} / \mu_{\mathrm{d} 3 \mathrm{EAN}}^{\prime}\right)^{1 / 2}=0.982$, where $\mu^{\prime}$ is the reduced mass, would be expected in the gasphase. For cation librations, IS $=\left(I_{\mathrm{EA}+} / I_{\mathrm{d} 3 \mathrm{EA}+}\right)^{1 / 2}$ should be 0.938 or 0.944 , depending on the chosen axis of inertia of the cation and the corresponding moment, $I .{ }^{77}$ The experimental ratios of the resonance frequencies are $\nu_{0, \mathrm{G} 2}^{\mathrm{d} 3 \mathrm{EAN}} / \nu_{0, \mathrm{G} 2}^{\mathrm{EAN}}=0.989$ and $\nu_{0, \mathrm{G} 3}^{\mathrm{d} 3 \mathrm{EAN}} / \nu_{0, \mathrm{G} 3}^{\mathrm{EAN}}=0.923$. These values differ significantly from each other and, more importantly, are gratifyingly close (given the assumptions involved) to the values of 0.982 predicted for H-bond stretching $\left(\mathrm{G}_{2}\right)$ and $0.938 / 0.944$ for cation libration $\left(\mathrm{G}_{3}\right)$. These assignments are also consistent with the the temperature dependences of $S_{\mathrm{G} 2}$ and $S_{\mathrm{G} 3}$ observed for EAN (Fig. 9a).

\subsection{PAN}

The high-frequency region of the DR spectra of PAN was fitted with the same model as that derived for EAN (Section 3.2): a damped harmonic oscillator, $\mathrm{DHO}_{1}$ (at $\sim 1.6 \mathrm{THz}$ ), three Gaussians, $\mathrm{G}_{1}(\sim 2.0 \mathrm{THz}), \mathrm{G}_{2}(\sim 4.8 \mathrm{THz})$, and $\mathrm{G}_{3}(\sim 6.5 \mathrm{THz})$ plus an additional mode $\left(\mathrm{DHO}_{2}\right.$ at $\left.9.0 \mathrm{THz}\right)$ associated with the intramolecular torsion of the propyl chain of $\mathrm{PrNH}_{3}^{+}$ with respect to the $\mathrm{NH}_{3}^{+}$group and which includes the intramolecular bending vibration of the propyl chain. ${ }^{13,78}$ 
It is not surprising (given their similar chemical structures) but is nevertheless gratifying that the model used to describe the DR spectra of EAN should also be applicable to PAN since it provides circumstantial evidence for the reasonableness of the EAN model. For example, the somewhat ad hoc introduction (Section 5.1) of an additional Gaussian $\left(\mathrm{G}_{3}\right)$ centered at $\sim 6 \mathrm{GHz}$ to best describe the DR spectrum of EAN at high frequencies, is supported by the clear-cut presence of a shoulder at $\sim 7 \mathrm{THz}$ in the DR spectrum of PAN in addition to the pronounced peak at $\sim 5 \mathrm{THz}$ described by $\mathrm{G}_{2}$ (compare Figs. 4 and 6 ). This provides strong support for more than one mode being responsible for the observed intensity in this region, consistent with the published FIR spectra. ${ }^{13,78}$

The amplitudes of the $\mathrm{DHO}_{1}, \mathrm{G}_{1}, \mathrm{G}_{2}$ and $\mathrm{G}_{3}$ modes for PAN are plotted in Fig. 12a. For all $S_{j}$, the effect of $T$ was the same as for EAN, ${ }^{79}$ again supporting the present mode assignment. Thus the resonance frequencies $\nu_{0, \mathrm{G} 1}, \nu_{0, \mathrm{G} 2}$ and $\nu_{0, \mathrm{G} 3}$ of PAN decrease with increasing $T$ (Fig. 12b) as observed for EAN (Fig. 9c) with the partial exception of $\nu_{0, \mathrm{G} 1}$. It is observed that $\nu_{0, \mathrm{G} 3}$ differs significantly (by $\sim 0.8 \mathrm{THz}$ ) between PAN and EAN, whereas $\nu_{0, \mathrm{G} 2}$ is almost the same (compare Figs.9c and 12b). Again such a difference is consistent with the assignment of $\mathrm{G}_{2}$ and $\mathrm{G}_{3}$ to different molecular-level processes. ${ }^{80}$

The present model provides a consistent description of the fast dynamics of EAN and PAN. In particular, it shows that the observed DR and OKE intensities at high frequencies $\left(\bar{\nu} \gtrsim 50 \mathrm{~cm}^{-1}\right)$ cannot be due to H-bond vibrations alone as has been proposed on the basis of FIR measurements and DFT calculations. ${ }^{13,78}$ Rather, there are also significant additional contributions from anion and cation librations (the $\mathrm{G}_{1}$ and $\mathrm{G}_{3}$ modes).

\subsection{Intermediate frequency range}

Sophisticated MD simulations and more accurate experimental studies will be required to fully understand the dynamics of PILs in the intermediate frequency range $(0.05 \lesssim \nu / \mathrm{THz}$ $\lesssim 1$ ) given the probable complexity of the contributing modes. The present DR and OKE spectra of EAN and PAN, like those observed for other ILs, ${ }^{17,81}$ show a finite but featureless 

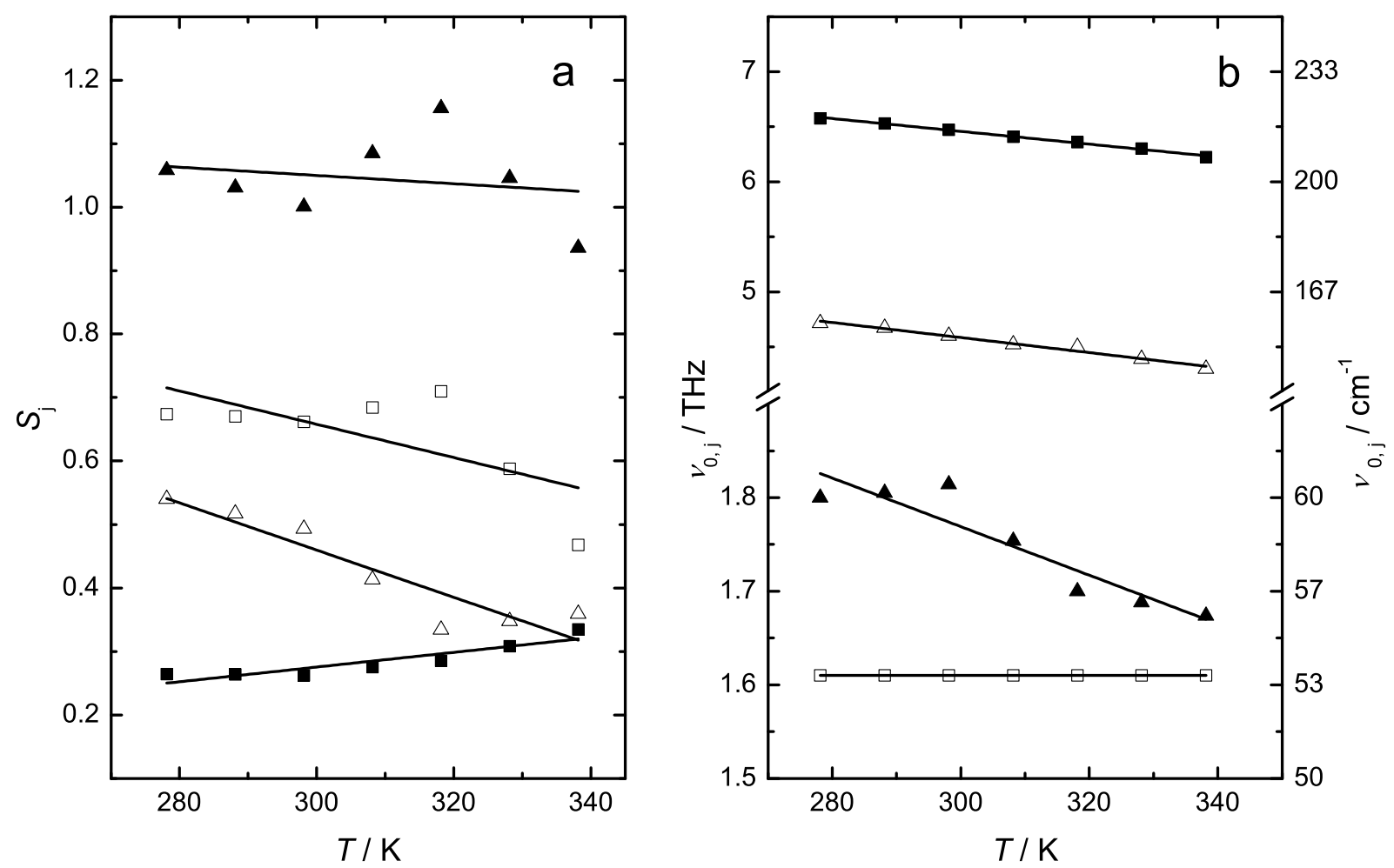

Figure 12: (a) Amplitudes, $S_{j}$, and (b) resonance frequencies, $\nu_{0, j}$, of the high-frequency resonance processes $\mathrm{DHO}_{1}(\square), \mathrm{G}_{1}(\boldsymbol{\Delta}), \mathrm{G}_{2}(\triangle)$ and $\mathrm{G}_{3}(\boldsymbol{\square})$ obtained from the fit of the DR spectra of PAN as a function of temperature. Solid lines are linear regressions of the data.

intensity over this range. This intensity reflects the strong coupling and/or overlap of the potentially numerous rotational and translational modes expected to occur in this region. Thus, the spectral contributions from the motions of individual molecular-level species, the oscillation of the cages formed by the surrounding neighbors, and the decay of these cages, blur into a continuous distribution of states. For PILs such as EAN and PAN fast proton transfers may also occur on this timescale. The combination of these factors makes a definitive separation and identification of the contributing processes a futile exercise at the present time. Accordingly, as already mentioned, the intensity of the OKE spectrum of EAN at intermediate frequencies was described by a constant-loss term of amplitude, $S_{\mathrm{CL}}$, which substitutes for all the possible contributions in this region. In contrast, in the DR spectrum of EAN and PAN, where this intensity is considerably smaller, it was more appropriate to subsume it in the CDi ( $\alpha$ relaxation) and $\mathrm{DHO}_{1}$ modes. 
For supercooled liquids, contributions on this time scale have been referred to as $\beta$ relaxations. ${ }^{82}$ Although the specific contributions to the $\beta$-relaxation are unknown, it seems likely that they originate, at least in part, from the motions of mobile clusters in an almost frozen environment, as proposed for glass-forming systems. ${ }^{82,83}$ Such motions are at least notionally comparable with the "cage rattling" translational motions suggested for imidazoliumbased ILs in the same spectral region. ${ }^{51}$

An additional contribution in this intermediate region might be analogous to the "frame tumbling" proposed for water. ${ }^{59}$ In contrast to librations this motion involves H-bond exchange and can be seen as a preliminary (faster) stage of the H-bond making and breaking associated with the $\alpha$-relaxation. Support for the presence of such a process in EAN comes from a MD simulation of the closely related methylammonium nitrate (MAN). ${ }^{84}$ This simulation predicts that $\mathrm{H}$-bond making and breaking takes place within $\sim 0.5 \mathrm{ps}$ while proton hopping happens within $\sim 3$ ps. Both processes are potential candidates for the observed intensity in the intermediate frequency range.

\section{Conclusions}

The combination of broadband DR and OKE spectroscopies covering the exceptionally wide frequency range of $0.2 \lesssim \nu / \mathrm{GHz} \lesssim 10000$ and (for most measurements) a temperature range of $5 \leq \vartheta /{ }^{\circ} \mathrm{C} \leq 65$ has provided detailed insights into the dynamics of the archetypal protic ionic liquid EAN and its congener PAN.

At lower frequencies $(\nu \lesssim 100 \mathrm{GHz})$ the $\alpha$-relaxation for EAN and PAN in the DR and OKE spectra could reasonably be assigned to the reorientation of the cations and the anions, respectively. A high degree of cooperativity in these motions was evident from the observed departures of the relaxation times from SED behavior and the similarities of the activation energies, $E_{\mathrm{A}}^{\mathrm{OKE}}$ and $E_{\mathrm{A}}^{\mathrm{DR}}$. This is consistent with the presence of strong and directed interionic interactions associated with strong H-bonding between the anions and cations, in line 
with previous studies of EAN mixtures with acetonitrile. ${ }^{85}$ Moreover, comparison of $\tau_{\alpha}^{\mathrm{DR}}$ for EAN with data from fs-IR spectroscopy ${ }^{56}$ indicates that the relaxation mechanism of the ions is not diffusive, but instead occurs through large-angle jump reorientations, with the jump angle being consistent with the pseudo-tetrahedral geometry of the alkylammonium ion. This suggests that in addition to the expected coulombic interactions, H-bonding between $\mathrm{EtNH}_{3}^{+}$and $\mathrm{NO}_{3}^{-}$plays an important role in determining the molecular dynamics of EAN. The negative temperature-dependence of $\mu_{\text {eff }}$ for EAN and PAN, also supports the picture of PIL relaxations as being highly cooperative.

Whilst the exact origins of the featureless intensity in the intermediate frequency region $(0.05 \lesssim \nu / \mathrm{THz} \lesssim 1)$ remain unclear, detailed insights into the high-frequency dynamics could still be obtained. Analysis of the DR and OKE spectra of EAN, supplemented by the DR spectra of PAN, as a function of temperature and OKE and DR spectra of $\mathrm{d}_{3}$-EAN at $25^{\circ} \mathrm{C}$ indicated that in the frequency range $1 \lesssim \nu / \mathrm{THz} \lesssim 10$, intermolecular (i.e., interionic) H-bond vibrations (bending and stretching) overlap with anion and cation librations. A summary of the present assignments is given in Table 1. The present results confirm in part the assignments of some of the features in the FIR spectra by Fumino et al. ${ }^{13}$ (Table 1) but also show that ion librations contribute significantly to those spectra. The present assignments are broadly supported by the isotopic shifts observed in the FIR spectra of EAN and $\mathrm{d}_{3}$-EAN.

While much has been learnt from the present investigation, further experimental studies using improved equipment and more sophisticated MD simulations that can quantitatively reproduce the DR and OKE spectra will be required for a fuller understanding of the dynamics of typical PILs such as EAN.

\section{Acknowledgement}

The authors thank Prof. W. Kunz (Universität Regensburg) for the provision of laboratory facilities and the Deutsche Forschungsgemeinschaft for the funding within the Priority 
Program 1191.

\section{Supporting Information Available}

Tables with physicochemical properties and relaxation parameters. This material is available free of charge via the Internet at http://pubs.acs.org.

\section{Notes and References}

(1) Greaves, T. L.; Drummond, C. J. Protic Ionic Liquids: Properties and Applications. Chem. Rev. 2008, 108, 206-237.

(2) Evans, D. F.; Chen, S.-H. Thermodynamics of Solution of Nonpolar Gases in a Fused Salt. "Hydrophobic Bonding" in a Nonaqueous System. J. Am. Chem. Soc. 1981, 103, $481-482$.

(3) Earle, M. J.; Esperanca, J. M. S. S.; Gilea, M. A.; Lopes, J. N. C.; Rebelo, L. P. N.; Magee, J. W.; Seddon, K. R.; Widegren, J. A. The Distillation and Volatility of Ionic Liquids. Nature 2006, 439, 831-834.

(4) MacFarlane, D. R.; Pringle, J. M.; Johansson, K. M.; Forsyth, S.; Forsyth, M. Lewis Base Ionic Liquids. Chem. Commun. 2006, 1905-1917.

(5) Susan, M. A. B. H.; Noda, A.; Mitsushima, S.; Watanabe, M. Brønsted Acid-base Ionic Liquids and their Use as New Materials for Anhydrous Proton Conductors. Chem. Commun. 2003, 938-939.

(6) Rana, U. A.; Forsyth, M.; MacFarlane, D. R.; Pringle, J. M. Toward Protic Ionic Liquid and Organic Plastic Crystal Electrolytes for Fuel Cells. Electrochimica Acta 2012, 84, $213-222$.

(7) Walden, P. Molecular Weights and Electrical Conductivities of Several Fused Salts. Bull. Acad. Impér. Soc. St. Petersburg 1914, 8, 405-422. 
(8) Zech, O.; Thomaier, S.; Kolodziejski, A.; Touraud, D.; Grillo, I.; Kunz, W. Ethylammonium Nitrate in High Temperature Stable Microemulsions. J. Colloid Interface Sci. 2010, 347, 227-232.

(9) Hayes, R.; Imberti, S.; Warr, G.; Atkin, R. Amphiphilicity Determines Nanostructure in Protic Ionic Liquids. Phys. Chem. Chem. Phys. 2011, 13, 3237-3247.

(10) Gontrani, L.; Bodo, E.; Triolo, A.; Leonelli, F.; D’Angelo, P.; Migliorati, V.; Caminiti, R. The Interpretation of Diffraction Patterns of Two Prototypical Protic Ionic Liquids: a Challenging Task for Classical Molecular Dynamics Simulations. J. Phys. Chem. B 2012, 116, 13024-13032.

(11) Umebayashi, Y.; Chung, W.; Mitsugi, T.; Fukuda, S.; Takeuchi, M.; Fujii, K.; Takamuku, T.; Kanzaki, R.; Ishiguro, S. Liquid Structure and the Ion-Ion Interactions of Ethylammonium Nitrate Ionic Liquid Studied by Large-Angle X-Ray Scattering and Molecular Dynamics Simulations. J. Comput. Chem. Jpn. 2008, 7, 125-134.

(12) Song, X.; Hamano, H.; Minofar, B.; Kanzaki, R.; Fujii, K.; Kameda, Y.; Kohara, S.; Watanabe, M.; Ishiguro, S.; Umebayashi, Y. Structural Heterogeneity and Unique Distorted Hydrogen Bonding in Primary Ammonium Nitrate Ionic Liquids Studied by High-Energy X-ray Diffraction Experiments and MD Simulation. J. Phys. Chem. B 2012, 116, 2801-2813.

(13) Fumino, K.; Wulf, A.; Ludwig, R. Hydrogen Bonding in Protic Ionic Liquids: Reminiscent of Water. Angew. Chem. Int. Ed. 2009, 48, 5184-5186.

(14) Fumino, K.; Reichert, E.; Wittler, K.; Hempelmann, R.; Ludwig, R. Low-Frequency Vibrational Modes of Protic Molten Salts and Ionic Liquids: Detecting and Quantifying Hydrogen Bonds. Angew. Chem. Int. Ed. 2012, 51, 6236-6240.

(15) Weingärtner, H.; Knocks, A.; Schrader, W.; Kaatze, U. Dielectric Spectroscopy of the 
Room Temperature Molten Salt Ethylammonium Nitrate. J. Phys. Chem. A 2001, $105,8646-8650$.

(16) Krüger, M.; Bründermann, E.; Funkner, S.; Weingärtner, H.; Havenith, M. Polarity Fluctuations of the Protic Ionic Liquid Ethylammonium Nitrate in the Terahertz Regime. J. Chem. Phys. 2010, 132, 101101.

(17) Turton, D. A.; Hunger, J.; Stoppa, A.; Hefter, G.; Thoman, A.; Walther, M.; Buchner, R.; Wynne, K. Dynamics of Imidazolium Ionic Liquids from a Combined Dielectric Relaxation and Optical Kerr Effect Study: Evidence for Mesoscopic Aggregation. J. Am. Chem. Soc. 2009, 131, 11140-11146.

(18) Turton, D. A.; Sonnleitner, T.; Ornter, A.; Walther, M.; Hefter, G.; Seddon, K. R.; Stana, S.; Plechkova, N.; Buchner, R.; Wynne, K. Structure and Dynamics in Protic Ionic Liquids: A Combined Optical Kerr-effect and Dielectric Relaxation Spectroscopy Study. Faraday Discuss. 2012, 154, 145-153.

(19) Evans, D. F.; Yamauchi, A.; Roman, R.; Casassa, E. Z. Micelle Formation in Ethylammonium Nitrate, a Low-Melting Fused Salt. J. Colloid Interface Sci. 1982, 88, 89-96.

(20) Sonnleitner, T.; Turton, D.; Waselikowski, S.; Hunger, J.; Stoppa, A.; Walther, M.; Wynne, K.; Buchner, R. Dynamics of RTILs: A Comparative Dielectric and OKE study. J. Mol. Liq. 2014, 192, 19-25.

(21) Barthel, J.; Buchner, R.; Eberspächer, P. N.; Münsterer, M.; Stauber, J.; Wurm, B. Dielectric Relaxation in Electrolyte Solutions. Recent Developments and Prospects. J. Mol. Liq. 1998, 78, 83-109.

(22) Schrödle, S.; Hefter, G.; Kunz, W.; Buchner, R. Effects of Nonionic Surfactant $\mathrm{C}_{12} \mathrm{E}_{5}$ on the Cooperative Dynamics of Water. Langmuir 2006, 22, 924-932. 
(23) Jepsen, P.; Fischer, B. M.; Thoman, A.; Helm, H.; Suh, J. Y.; Lopez, R.; Haglund, R. F. Metal-insulator Phase Transition in a $\mathrm{VO}_{2}$ Thin Film Observed with Terahertz Spectroscopy. Phys. Rev. B 2006, 74, 205103.

(24) Hunger, J.; Stoppa, A.; Thoman, A.; Walther, M.; Buchner, R. Broadband Dielectric Response of Dichloromethane. Chem. Phys. Lett. 2009, 471, 85-91.

(25) (a) Böttcher, C. F. J., Ed. Theory of Electric Polarization, Vol. 1; Elsevier: Amsterdam, 1973; (b) Böttcher, C. F. J., Bordewijk, P., Eds. Theory of Electric Polarization, Vol. 2; Elsevier: Amsterdam, 1978.

(26) Buchner, R.; Hefter, G. Interactions and Dynamics in Electrolyte Solutions by Dielectric Spectroscopy. Phys. Chem. Chem. Phys. 2009, 11, 8984-8999.

(27) Fecko, C. J.; Eaves, J. D.; Tomakoff, A. Isotropic and Anisotropic Raman Scattering from Molecular Liquids Measured by Spatially Masked Optical Kerr Effect Spectroscopy. J. Chem. Phys. 2002, 117, 1139-1154.

(28) Shaukat, S.; Buchner, R. Densities, Viscosities [from (278.15 to 318.15) K], and Electrical Conductivities (at 298.15 K) of Aqueous Solutions of Choline Chloride and ChloroCholine Chloride. J. Chem. Eng. Data 2011, 56, 4944-4949.

(29) Greaves, T. L.; Weerawardena, A.; Fong, C.; Krodkiewska, I.; Drummond, C. J. Protic Ionic Liquids: Solvents with Tunable Phase Behavior and Physicochemical Properties. J. Phys. Chem. B 2006, 110, 22479-22487.

(30) Poole, C. F.; Kersten, B. R.; Ho, S. S.; Coddens, M. E.; Furton, K. G. Organic Salts, Liquid at Room Temperature, as Mobile Phases in Liquid Chromatography. J. Chromatogr. 1986, 352, 407-425.

(31) Shetty, P. H.; Youngberg, P. J.; Kersten, B. R.; Poole, C. F. Solvent Properties of 
Liquid Organic Salts used as Mobile Phases in Microcolumn Reversed-Phase Liquid Chromatography. J. Chromatogr. 1987, 411, 61-79.

(32) Atkin, R.; Warr, G. The smallest Amphiphiles: Nanostructure in Protic RoomTemperature Ionic Liquids with Short Alkyl Groups. J. Phys. Chem. B 2008, 112, $4146-4166$.

(33) Belieres, J.-P.; Angell, C. A. Protic Ionic Liquids: Preparation, Characterization, and Proton Free Energy Level Representation. J. Phys. Chem. B 2007, 111, 4926-4937.

(34) Oleinikova, A.; Bonetti, M. Critical Behavior of the Electrical Conductivitiy of Concentrated Electrolytes: Ethylammonium Nitrate in n-Octanol Binary Mixtures. J. Solution Chem. 2002, 31, 397-413.

(35) Ferry, J. D. Viscoelastic Properties of Polymers; Wiley: New York, 1980.

(36) Angell, C. A. Relaxation in Liquids, Polymers and Plastic Crystals - Strong/fragile Patterns and Problems. J. Non-Cryst. Solids 1991, 131-133, 13-31.

(37) Smith, J. A.; Webber, G. B.; Warr, G. G.; Atkin, R. Rheology of Protic Ionic Liquids and Their Mixtures. J. Phys. Chem. B 2013, 117, 13930-13935.

(38) Bouzón Capelo, S.; Méndez-Morales, T.; Carrete, J.; López Lago, E.; Vila, J.; Cabeza, O.; Rodriguez, J. R.; Turmine, M.; Varela, L. M. Effect of Temperature and Cationic Chain Length on the Physical Properties of Ammonium Nitrate-Based Protic Ionic Liquids. J. Phys. Chem. B 2012, 116, 11302-11312.

(39) Schröder, C.; Steinhauser, O. In Computational dielectric spectroscopy of charged, dipolar systems; Grünenberg, J., Ed.; Wiley-VCH: Weinheim, Germany, 2010.

(40) Schröder, C.; Haberler, M.; Steinhauser, O. On the Computation and Contribution of Conductivity in Molecular Ionic Liquids. J. Chem. Phys. 2008, 128, 134501. 
(41) Schröder, C. Collective Translational Motions and Cage Relaxations in Molecular Ionic Liquids. J. Chem. Phys. 2011, 135, 024502.

(42) Ishida, T.; Nishikawa, K.; Shirota, H. Atom Substitution Effects of $\left[\mathrm{XF}_{6}\right]^{-}$in Ionic Liquids. 2. Theoretical Study. J. Phys. Chem. B 2009, 113, 9840-9851.

(43) Ishida, T.; Shirota, H. Dicationic versus Monocationic Ionic Liquids: Distinctive Ionic Dynamics and Dynamical Heterogeneity. J. Phys. Chem. B 2009, 117, 1136-1150.

(44) Schröder, C.; Sonnleitner, T.; Buchner, R.; Steinhauser, O. The influence of polarizability on the dielectric spectrum of the ionic liquid 1-ethyl-3-imidazolium triflate. Phys. Chem. Chem. Phys. 2011, 13, 12240-12248.

(45) Turton, D.; Wynne, K. Structural Relaxation in the Hydrogen-bonding Liquids Nmethylacetamide and Water Studied by Optical Kerr Effect Spectroscopy. J. Chem. Phys. 2008, 128, 154516.

(46) Chalmers, J. M.; Griffiths, P. R. Handbook of Vibrational Spectroscopy; Wiley-VCH: Weinheim, 2001.

(47) Lozier, D. M., Boisvert, R. F., Clark, C. W., Eds. NIST Handbook of Mathematical Functions; Cambridge Univ. Press: Cambridge, 2010.

(48) Hunger, J.; Stoppa, A.; Schrödle, S.; Hefter, G.; Buchner, R. Temperature Dependence of the Dielectric Properties and Dynamics of Ionic Liquids. Chem. Phys. Chem. 2009, $10,723-733$.

(49) Cang, H.; Novikov, N.; Fayer, M. D. Logarithmic Decay of the Orientational Correlation Function in Supercooled Liquids on the ps to ns Time Scale. J. Chem. Phys. 2003, 118, $2800-2807$.

(50) Buchner, R.; Chen, T.; Hefter, G. Complexity in Simple Electrolyte Solutions: Ion Pairing in $\mathrm{MgSO}_{4}(\mathrm{aq})$. J. Phys. Chem. B 2004, 108, 2365-2375. 
(51) Turton, D.; Corsaro, C.; Candelaresi, M.; Brownlie, A.; Seddon, K. R.; Mallamace, F.; Wynne, K. The Structure and Terahertz Dynamics of Water Confined in Nanoscale Pools in Salt Solutions. Faraday Discuss. 2011, 150, 493-504.

(52) Huang, M.-M.; Jiang, Y.; Sasisanker, P.; Driver, G. W.; Weingärtner, H. Static Relative Dielectric Permittivities of Ionic Liquids at $25^{\circ} \mathrm{C}$. J. Chem. Eng. Data 2011, 56, 14941499.

(53) Kremer, F.; Schönhals, A. Broadband Dielectric Spectroscopy; Springer, Berlin, 2003.

(54) Salvador, P.; Curtis, J. E.; Tobias, D. J.; Jungwirth, P. Polarizability of the Nitrate Anion and its Solvation at the Air/water Interface. Phys. Chem. Chem. Phys. 2003, $5,3752-3757$.

(55) Stewart, J. J. P. M. Stewart Computational Chemistry; Colorado Springs, CO, USA, 2009.

(56) Hunger, J.; Sonnleitner, T.; Liu, L.; Buchner, R.; Bonn, M.; Bakker, H. J. HydrogenBond Dynamics in a Protic Ionic Liquid: Evidence of Large-Angle Jumps. J. Phys. Chem. Lett. 2012, 3, 3034-3038.

(57) Note that for comparison with the fs-IR relaxation times, the Cole-Davidson relaxation times, $\tau_{\alpha}^{\mathrm{DR}}$, obtained from the dielectric fit were converted into relaxation times of the $\alpha$ peak maximum, $\tau_{\alpha, \max }^{\mathrm{DR}}$, using the eq 4 given in Ref. 86 .

(58) Shim, Y.; Kim, H. Dielectric Relaxation, Ion Conductivity, Solvent Rotation, and Solvation Dynamics in Room-Temperature Ionic Liquid. J. Phys. Chem. B 2008, 112, $11028-11038$.

(59) Laage, D.; Stirnemann, G.; Sterpone, F.; Rey, R.; Hynes, J. T. Reorientation and Allied Dynamics in Water and Aqueous Solutions. Annu. Rev. Phys. Chem. 2011, 62, $395-416$. 
(60) Ohmine, I.; Tanaka, H. Fluctuation, Relaxations, and Hydration in Liquid Water. Hydrogen-Bond Rearrangement Dynamics. Chem. Rev. 1993, 93, 2545-2566.

(61) Izgorodina, E. I.; Forsyth, M.; MacFarlane, D. R. On the Components of the Dielectric Constants of Ionic Liquids: Ionic Polarization? Phys. Chem. Chem. Phys. 2009, 11, $2452-2458$.

(62) Barthel, J.; Hetzenauer, H.; Buchner, R. Dielectric Relaxation of Aqueous Electrolyte Solutions. II. Ion-Pair Relaxation of 1:2, 2:1, and 2:2 Electrolytes. Ber. Bunsen-Ges. Phys. Chem. 1992, 96, 1424-1432.

(63) Scholte, T. A Contribution to the Theory of the Dielectric Constant of Polar Liquids. Physica 1949, 15, 437-449.

(64) The difference between $\mu_{\mathrm{eff}}$ and $\mu_{\mathrm{app}}$ of PAN can be explained by the fact that in the liquid phase the pivot does not necessarily coincide with the center of gravity but more likely with the center of hydrodynamic stress; ${ }^{62}$ the higher flexibility of $\mathrm{PrNH}_{3}^{+}$may also play a role.

(65) Heisler, I. A.; Meech, S. R. Polarization-Resolved Ultrafast Polarizability Relaxation in Polar Aromatic Liquids. J. Phys. Chem. B 2008, 112, 12976-12984.

(66) Fukasawa, T.; Sato, T.; Watanabe, J.; Hama, Y.; Kunz, W.; Buchner, R. Relation between Dielectric and Low-Frequency Raman Spectra of Hydrogen-Bond Liquids. Phys. Rev. Lett. 2005, 95, 197802.

(67) Winkler, K.; Lindner, J.; Vöhringer, P. Phys. Chem. Chem. Phys. 2002, 4, 2144-2155.

(68) Bartoli, F. J.; Litovitz, T. A. Raman Scattering: Orientational Motions in Liquids. J. Chem. Phys. 1972, 56, 413-425.

(69) Zelsmann, H. R. Temperature Dependence of the Optical Constants for Liquid $\mathrm{H}_{2} \mathrm{O}$ and $\mathrm{D}_{2} \mathrm{O}$ in the Far IR Region. J. Mol. Struct. 1995, 350, 95-114. 
(70) Castner, E. W.; Maroncelli, M. Solvent Dynamics Derived from Optical Kerr Effect, Dielectric Dispersion, and Time-Resolved Stokes Shift Measurements: An Empirical Comparison. J. Mol. Liq. 1998, 77, 1-36.

(71) Palombo, F.; Paolantoni, M.; Sassi, P.; Morresi, A.; Giorgini, M. G. Molecular Dynamics of Liquid Acetone Determined by Depolarized Rayleigh and Low-frequency Raman Scattering Spectroscopy. Phys. Chem. Chem. Phys. 2011, 13, 16197-16207.

(72) Shirota, H.; Funston, A. M.; Wishart, J. F.; Castner, E. W. Ultrafast Dynamics of Pyrrolidinium Cation Ionic Liquids. J. Chem. Phys. 2005, 122, 184512.

(73) Shirota, H.; Fukazawa, H.; Fujisawa, T.; Wishart, J. F. Heavy Atom Substitution Effects in Non-Aromatic Ionic Liquids: Ultrafast Dynamics and Physical Properties. J. Phys. Chem. B 2010, 114, 9400-9412.

(74) Amo, Y.; Tominaga, Y. Low-Frequency Raman Study of Water Isotopes. Physica A 2000, 276, 401-412.

(75) Gaiduk, V. I.; Nielsen, O. F.; Crothers, D. S. F. Molecular Theory of Low-frequency Raman Spectrum of Water in the Translational-band Region. J. Mol. Liq. 2008, 137, 92-103.

(76) Brubach, J.-B.; Mermet, A.; Filabozzi, A.; Gerschel, A.; Roy, P. Signatures of the Hydrogen Bonding in the Infrared Bands of Water. J. Chem. Phys. 2005, 122, 184509.

(77) The relevant moments of inertia determined by MOPAC ${ }^{55}$ for EAN and $\mathrm{d}_{3}$-EAN (assuming rigid molecules) are $I_{\mathrm{B}}=100.6035 \times 10^{-47} \mathrm{~kg} \mathrm{~m}^{2} \& I_{\mathrm{C}}=114.4250 \times 10^{-47} \mathrm{~kg} \mathrm{~m}^{2}$ and $I_{\mathrm{B}}=114.3995 \times 10^{-47} \mathrm{~kg} \mathrm{~m}^{2} \& I_{\mathrm{C}}=128.4374 \times 10^{-47} \mathrm{~kg} \mathrm{~m}^{2}$, respectively. Indices 'B' and ' $\mathrm{C}$ ' denote the principle axes of inertia perpendicular to the dipole vector.

(78) Fumino, K.; Wulf, A.; Ludwig, R. The Potential Role of Hydrogen Bonding in Aprotic and Protic Ionic Liquids. Phys. Chem. Chem. Phys. 2009, 11, 8790-8794. 
(79) Except perhaps $S_{\mathrm{G} 1}$, which increases with increasing $T$ for EAN (Fig. 9a, $\mathbf{\Delta}$ ) but is almost constant for PAN (Fig. 12a, $\mathbf{\Delta}$ ). However, this difference may result from the considerable scatter in the THz-TDS data (Fig. 4) and the absence of OKE data to fix $\nu_{0, \mathrm{G} 1}$.

(80) Note, however, that the harmonic oscillator model would predict $\nu_{0, \mathrm{G} 3}(\mathrm{EAN})>$ $\nu_{0, \mathrm{G} 3}(\mathrm{PAN})$, opposite to the present results. This possibly reflects the effects of the greater length (size) and flexibility of the propyl chain on the librational cage. In contrast $\mathrm{G}_{2}$, assigned here to H-bond stretching, would be expected to be less affected when Et is replaced by $\operatorname{Pr}$, because of its translational character.

(81) Stoppa, A.; Hunger, J.; Buchner, R.; Hefter, G.; Thoman, A.; Helm, H. Interactions and Dynamics in Ionic Liquids. J. Phys. Chem. B 2008, 112, 4854-4858.

(82) Götze, W.; Sjogren, L. Relaxation Processes in Supercooled Liquids. Rep. Prog. Phys. 1992, 55, 241-376.

(83) Johari, G. P.; Whalley, E. Dielectric Properties of Glycerol in the Range 0.1-105 Hz, 218-357 K, 0-53 kb. Faraday Symp. Chem. Soc. 1972, 6, 23-42.

(84) Zahn, S.; Thar, J.; Kirchner, B. Structure and Dynamics of the Protic Ionic Liquid Monomethylammonium Nitrate $\left(\left[\mathrm{CH}_{3} \mathrm{NH}_{3}\right]\left[\mathrm{NO}_{3}\right]\right)$ from Ab Initio Molecular Dynamics Simulations. J. Chem. Phys. 2010, 132, 124506.

(85) Sonnleitner, T.; Nikitina, V.; Nazet, A.; Buchner, R. Do H-Bonds Explain Strong Ion Aggregation in Ethylammonium Nitrate + Acetonitrile Mixtures? Phys. Chem. Chem. Phys. 2013, 15, 18445-18452.

(86) Diaz-Calleja, R. Comment on the Maximum in the Loss Permittivity for the HavriliakNegami Equation. Macromolecules 2000, 33, 8924. 


\section{TOC Fig.}

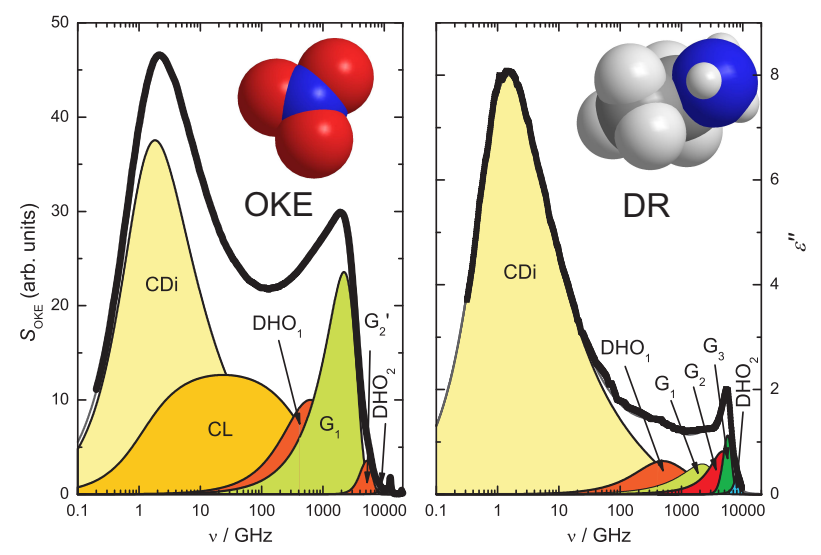

This material is available free of charge via the Internet at http://pubs.acs.org/. 


\title{
Supplementary Information
}

\section{An Ultra-Broadband Dielectric and Optical} Kerr-Effect Study of the Ionic Liquids Ethyland Propylammonium Nitrate

\author{
Thomas Sonnleitner, ${ }^{\dagger}$ David A. Turton, ${ }^{\ddagger}$ Glenn Hefter, ${ }^{\circledR}$ Alexander Ortner,,${ }^{\S}$ Stefan \\ Waselikowski, ${ }^{\S}$ Markus Walther, ${ }^{\S}$ Klaas Wynne, ${ }^{\ddagger}$ and Richard Buchner ${ }^{*} \dagger$ \\ Institut für Physikalische und Theoretische Chemie, Universität Regensburg, D-93040 \\ Regensburg, Germany, School of Chemistry, University of Glasgow, Glasgow, G12 8QQ \\ United Kingdom, Chemistry Department, Murdoch University, Murdoch, W.A. 6150, \\ Australia, and Department of Molecular and Optical Physics, Albert-Ludwigs-Universität \\ Freiburg, 79104 Freiburg, Germany
}

E-mail: Richard.Buchner@chemie.uni-regensburg.de

\footnotetext{
${ }^{*}$ To whom correspondence should be addressed

${ }^{\dagger}$ Institut für Physikalische und Theoretische Chemie, Universität Regensburg, D-93040 Regensburg, Germany

${ }^{\ddagger}$ School of Chemistry, University of Glasgow, Glasgow, G12 8QQ United Kingdom

${ }^{\top}$ Chemistry Department, Murdoch University, Murdoch, W.A. 6150, Australia

${ }^{\S}$ Department of Molecular and Optical Physics, Albert-Ludwigs-Universität Freiburg, 79104 Freiburg, Germany
} 


\section{Densities, Viscosities and Conductivities}

Table S1: Temperature dependence of density, $\rho$, and viscosity, $\eta$, of EAN and PAN and electrical conductivity, $\kappa$, of EAN.

\begin{tabular}{ccccccc} 
& \multicolumn{3}{c}{ EAN } & & \multicolumn{2}{c}{ PAN } \\
\cline { 2 - 4 } \cline { 6 - 7 }$T / \mathrm{K}$ & $\rho / \mathrm{gcm}^{-3}$ & $\eta / \mathrm{mPas}$ & $\kappa / \mathrm{S} \mathrm{m}^{-1}$ & & $\rho / \mathrm{g} \mathrm{cm}^{-3}$ & $\eta / \mathrm{mPas}$ \\
\hline 268.15 & - & - & 0.693 & & - & - \\
278.15 & - & 86.5 & 1.09 & & 1.16319 & 152 \\
288.15 & 1.21657 & 56.0 & 1.61 & & 1.15685 & 92.1 \\
298.15 & 1.21027 & 38.6 & 2.24 & & 1.15063 & 60.2 \\
308.15 & 1.20411 & 27.9 & 2.98 & & 1.14454 & 41.3 \\
318.15 & 1.19807 & 21.0 & 3.38 & & 1.13855 & 29.8 \\
328.15 & 1.19213 & 16.4 & 4.78 & & 1.13266 & 22.4 \\
338.15 & 1.18631 & 13.1 & 5.82 & & 1.12686 & 17.5 \\
348.15 & - & 10.5 & 6.93 & & - & 13.9 \\
358.15 & - & 8.70 & 8.12 & & - & 11.4 \\
368.15 & - & 7.39 & 9.37 & & - & 9.48 \\
378.15 & - & 6.33 & 10.7 & & - & 7.65 \\
388.15 & - & 5.50 & 12.0 & & - & 6.53 \\
398.15 & - & 4.82 & 13.4 & & -
\end{tabular}

Table S2: VFT parameters for viscosity, $\eta$, and resistivity, $\kappa^{-1}$, of EAN and PAN of the present work (p.w.) and from the literature.

\begin{tabular}{|c|c|c|c|c|c|c|c|}
\hline \multirow{3}{*}{ VFT parameters } & \multicolumn{4}{|c|}{$\mathrm{EAN}$} & \multicolumn{3}{|c|}{ PAN } \\
\hline & \multicolumn{2}{|c|}{$\eta(T)$} & \multicolumn{2}{|c|}{$\kappa^{-1}(T)$} & \multicolumn{2}{|c|}{$\eta(T)$} & \multirow{2}{*}{$\frac{\kappa^{-1}(T)}{\text { lit. }^{4}}$} \\
\hline & p.w. & lit. $^{1}$ & p.w. & lit. $^{2}$ & p.w. & lit. ${ }^{3}$ & \\
\hline $\ln Y_{0}$ & -8.47 & -8.84 & -5.10 & -5.10 & -8.62 & -7.85 & -4.16 \\
\hline$B_{\mathrm{VFT}} / \mathrm{K}$ & 786.4 & 903.5 & 601.4 & 599.6 & 855.6 & 676 & 485.7 \\
\hline$T_{0} / \mathrm{K}$ & 147.5 & 133.6 & 158.2 & 157.2 & 150.7 & 169 & 181.9 \\
\hline
\end{tabular}




\section{Spectroscopic Model Selection}

Table S3: OKE fit parameters of EAN as a function of temperature and of $\mathrm{d}_{3}-\mathrm{EAN}$ at $25^{\circ} \mathrm{C}$ : relaxation and resonance amplitudes, $S_{j}$, relaxation times, $\tau_{\alpha}$, Cole-Davidson parameter, $\beta$, resonance frequencies, $\nu_{0, j}$, damping constants, $\gamma_{j}$, and reduced error function, $\chi_{\mathrm{r}}^{2}{ }^{a}$

\begin{tabular}{ccccccccc} 
IL & $T / \mathrm{K}$ & $S_{\alpha}$ & $\beta$ & $\tau_{\alpha}$ & $S_{\mathrm{CL}}$ & $S_{\mathrm{DHO} 1}$ & $\gamma_{\mathrm{DHO} 1}$ & $\nu_{0, \mathrm{DHO} 1}$ \\
\hline \multirow{2}{*}{ EAN } & 278.15 & 111 & 0.569 & 291 & 51.8 & 17.5 & 4.83 & 1.67 \\
& 288.15 & 108 & 0.562 & 197 & 51.9 & 18.0 & 4.54 & 1.62 \\
& 298.15 & 95.7 & 0.569 & 136 & 51.7 & 17.4 & 4.09 & 1.51 \\
& 308.15 & 89.5 & 0.597 & 96.1 & 53.3 & 17.4 & 4.24 & 1.53 \\
& 318.15 & 81.0 & 0.593 & 73.0 & 52.6 & 17.3 & 4.10 & 1.47 \\
& 328.15 & 74.6 & 0.593 & 58.6 & 52.6 & 17.3 & 4.20 & 1.49 \\
- & 338.15 & 70.6 & 0.594 & 46.4 & 50.7 & 17.6 & 4.18 & 1.48 \\
d ${ }_{3}$-EAN & 298.15 & 106 & $0.569^{c}$ & 148 & 70.0 & 22.2 & $4.09^{c}$ & 1.50 \\
\hline
\end{tabular}

\begin{tabular}{ccccccccccc} 
IL & $T / \mathrm{K}$ & $S_{\mathrm{G} 1}$ & $\gamma_{\mathrm{G} 1}$ & $\nu_{0, \mathrm{G} 1}$ & $S_{\mathrm{G} 2^{\prime}}$ & $\gamma_{\mathrm{G}^{\prime}}$ & $\nu_{0, \mathrm{G} 2^{\prime}}$ & $S_{\mathrm{DHO} 2}^{b}$ & $\gamma_{\mathrm{DHO} 2}^{b}$ & $\chi_{\mathrm{r}}^{2}$ \\
\hline \multirow{3}{*}{ EAN } & 278.15 & 26.0 & 1.23 & 2.22 & 1.24 & 1.12 & 5.17 & 0.05 & 1.5 & 0.0493 \\
& 288.15 & 26.4 & 1.24 & 2.19 & 1.31 & 1.16 & 5.19 & 0.05 & 1.5 & 0.0396 \\
& 298.15 & 26.7 & 1.25 & 2.17 & 1.36 & 1.17 & 5.15 & 0.05 & 1.5 & 0.0291 \\
& 308.15 & 26.7 & 1.25 & 2.13 & 1.52 & 1.24 & 5.11 & 0.05 & 1.5 & 0.0225 \\
& 318.15 & 27.4 & 1.27 & 2.10 & 1.51 & 1.25 & 5.12 & 0.05 & 1.5 & 0.0258 \\
& 328.15 & 26.8 & 1.26 & 2.08 & 1.62 & 1.28 & 5.04 & 0.05 & 1.5 & 0.0279 \\
& 338.15 & 26.6 & 1.26 & 2.05 & 1.72 & 1.31 & 4.98 & 0.05 & 1.5 & 0.0276 \\
\hline
\end{tabular}

$\begin{array}{lllllllllll}\mathrm{d}_{3} \text {-EAN } & 298.15 & 36.3 & 1.25^{c} & 2.15 & 2.06 & 1.17^{c} & 5.12 & 0.05 & 1.5 & 0.056\end{array}$ ${ }^{a}$ Units: $\tau_{\alpha}$ in ps, $\nu_{0, j}$ in THz, $\gamma_{j}$ in THz; ${ }^{b}$ Parameter fixed; ${ }^{c}$ Parameter fixed to that of EAN. 
Table S4: DR fit parameters of EAN as a function of temperature and of $\mathrm{d}_{3}$-EAN at $25^{\circ} \mathrm{C}$ : static permittivity, $\varepsilon_{\mathrm{s}}$, relaxation and resonance amplitudes, $S_{j}$, relaxation time, $\tau_{\alpha}$, ColeDavidson parameter, $\beta$, resonance frequencies, $\nu_{0, j}$, damping constants, $\gamma_{j}$, high frequency limit of $\varepsilon^{\prime}, \varepsilon_{\infty}$, and reduced error function, $\chi_{\mathrm{r}}^{2}{ }^{a}$

\begin{tabular}{cccccccccccc} 
IL & $T / \mathrm{K}$ & $\varepsilon_{\mathrm{s}}$ & $S_{\alpha}$ & $\beta^{b}$ & $\tau_{\alpha}$ & $S_{\mathrm{DHO} 1}$ & $\gamma_{\mathrm{DHO} 1}^{b}$ & $\nu_{0, \mathrm{DHO} 1}^{c}$ & $S_{\mathrm{G} 1}$ & $\gamma_{\mathrm{G} 1}$ & $\nu_{0, \mathrm{G} 1}$ \\
\hline \multirow{3}{*}{ EAN } & 278.15 & 30.0 & 24.5 & 0.5 & 354 & 1.72 & 5.0 & 1.67 & 0.238 & 0.765 & 2.22 \\
& 288.15 & 29.1 & 23.7 & 0.5 & 250 & 1.44 & 5.0 & 1.62 & 0.462 & 1.05 & $2.19^{c}$ \\
& 298.15 & 27.3 & 21.9 & 0.5 & 185 & 1.15 & 5.0 & 1.51 & 0.670 & 1.34 & $2.17^{c}$ \\
& 308.15 & 26.3 & 20.9 & 0.5 & 143 & 1.19 & 5.0 & 1.53 & 0.733 & 1.65 & $2.13^{c}$ \\
& 318.15 & 24.5 & 19.2 & 0.5 & 107 & 1.23 & 5.0 & 1.47 & 0.756 & 1.93 & $2.10^{c}$ \\
& 328.15 & 23.6 & 18.3 & 0.5 & 83.3 & 1.12 & 5.0 & 1.49 & 0.802 & 1.96 & $2.08^{c}$ \\
& 338.15 & 22.1 & 16.9 & 0.5 & 69.8 & 0.999 & 5.0 & 1.48 & 0.841 & 1.98 & $2.05^{c}$ \\
$\mathrm{~d}_{3}$-EAN & 298.15 & 27.2 & 21.8 & 0.5 & 192 & 1.34 & 5.0 & 1.51 & 0.641 & $1.34^{d}$ & $2.17^{c}$ \\
\hline
\end{tabular}

\begin{tabular}{cccccccccccc} 
IL & $T / \mathrm{K}$ & $S_{\mathrm{G} 2}$ & $\gamma_{\mathrm{G} 2}$ & $\nu_{0, \mathrm{G} 2}$ & $S_{\mathrm{G} 3}$ & $\gamma_{\mathrm{G} 3}$ & $\nu_{0, \mathrm{G} 3}$ & $S_{\mathrm{DHO} 2}^{b}$ & $\gamma_{\mathrm{DHO} 2}^{b}$ & $\varepsilon_{\infty}$ & $\chi_{\mathrm{r}}^{2} \cdot 10^{4}$ \\
\hline \multirow{3}{*}{ EAN } & 278.15 & 0.714 & 1.58 & 4.85 & 0.167 & 0.722 & 5.81 & 0.08 & 1.9 & 2.58 & 51 \\
& 288.15 & 0.695 & 1.72 & 4.78 & 0.220 & 0.799 & 5.74 & 0.08 & 1.9 & 2.57 & 165 \\
& 298.15 & 0.638 & 1.83 & 4.69 & 0.290 & 0.892 & 5.65 & 0.08 & 1.9 & 2.55 & 76 \\
& 308.15 & 0.600 & 1.96 & 4.65 & 0.312 & 0.915 & 5.65 & 0.08 & 1.9 & 2.49 & 78 \\
& 318.15 & 0.543 & 2.07 & 4.59 & 0.328 & 0.914 & 5.61 & 0.08 & 1.9 & 2.42 & 161 \\
& 328.15 & 0.458 & 2.09 & 4.54 & 0.381 & 0.974 & 5.58 & 0.08 & 1.9 & 2.44 & 291 \\
& 338.15 & 0.379 & 2.12 & 4.47 & 0.428 & 1.01 & 5.54 & 0.08 & 1.9 & 2.45 & 102 \\
\hline
\end{tabular}

$\begin{array}{llllllllllll}\mathrm{d}_{3} \text {-EAN } & 298.15 & 0.701 & 1.83^{d} & 4.63 & 0.265 & 0.892^{d} & 5.21 & 0.035 & 1.9 & 2.40 & 84\end{array}$

${ }^{a}$ Units: $\tau_{\alpha}$ in ps, $\nu_{0, j}$ in THz, $\gamma_{j}$ in THz; ${ }^{b}$ Parameter fixed; ${ }^{c}$ Parameter fixed to that of OKE fit; ${ }^{d}$ Parameter fixed to that of EAN; Italic values were interpolated (see text). 
Table S5: DR fit parameters of PAN as a function of temperature: static permittivity, $\varepsilon_{\mathrm{s}}$, relaxation and resonance amplitudes, $S_{j}$, relaxation time, $\tau_{\alpha}$, Cole-Davidson parameter, $\beta$, resonance frequencies, $\nu_{0, j}$, damping constants, $\gamma_{j}$, high frequency limit of $\varepsilon^{\prime}$, $\varepsilon_{\infty}$, and reduced error function, $\chi_{\mathrm{r}}^{2}{ }^{a}$

\begin{tabular}{ccccccccccc}
$T / \mathrm{K}$ & $\varepsilon_{\mathrm{S}}$ & $S_{\alpha}$ & $\beta$ & $\tau_{\alpha}$ & $S_{\mathrm{DHO} 1}$ & $\gamma_{\mathrm{DHO} 1}^{b}$ & $\nu_{0, \mathrm{DHO} 1}^{b}$ & $S_{\mathrm{G} 1}$ & $\gamma_{\mathrm{G} 1}$ & $\nu_{0, \mathrm{G} 1}$ \\
\hline 278.15 & 26.3 & 21.6 & 0.394 & 1536 & 0.674 & 5.0 & 1.61 & 1.06 & $1.61^{b}$ & $1.80^{b}$ \\
288.15 & 25.2 & 20.6 & 0.391 & 988 & 0.670 & 5.0 & 1.61 & 1.03 & 1.71 & 1.81 \\
298.15 & 23.4 & 18.8 & 0.398 & 628 & 0.662 & 5.0 & 1.61 & 1.00 & 1.81 & 1.81 \\
308.15 & 23.8 & 19.2 & 0.409 & 483 & 0.684 & 5.0 & 1.61 & 1.08 & 1.91 & 1.75 \\
318.15 & 21.6 & 17.0 & 0.391 & 342 & 0.710 & 5.0 & 1.61 & 1.16 & $2.00^{b}$ & $1.70^{b}$ \\
328.15 & 22.5 & 18.2 & 0.370 & 325 & 0.588 & 5.0 & 1.61 & 1.05 & 2.06 & 1.69 \\
338.15 & 20.0 & 15.6 & $0.400^{b}$ & 179 & 0.468 & 5.0 & 1.61 & 0.936 & 2.12 & 1.67 \\
\hline
\end{tabular}

\begin{tabular}{ccccccccccc}
$T / \mathrm{K}$ & $S_{\mathrm{G} 2}$ & $\gamma_{\mathrm{G} 2}$ & $\nu_{0, \mathrm{G} 2}$ & $S_{\mathrm{G} 3}$ & $\gamma_{\mathrm{G} 3}$ & $\nu_{0, \mathrm{G} 3}$ & $S_{\mathrm{DHO} 2}^{b}$ & $\gamma_{\mathrm{DHO} 2}^{b}$ & $\varepsilon_{\infty}$ & $\chi_{\mathrm{r}}^{2} \cdot 10^{4}$ \\
\hline 278.15 & 0.540 & 1.17 & 4.72 & 0.264 & 1.14 & 6.58 & 0.05 & 1.8 & 2.05 & 104 \\
288.15 & 0.517 & 1.21 & 4.67 & 0.264 & 1.16 & 6.53 & 0.05 & 1.8 & 2.11 & 210 \\
298.15 & 0.493 & 1.24 & 4.60 & 0.262 & 1.18 & 6.47 & 0.05 & 1.8 & 2.17 & 156 \\
308.15 & 0.413 & 1.14 & 4.52 & 0.275 & 1.17 & 6.41 & 0.05 & 1.8 & 2.07 & 57 \\
318.15 & 0.334 & 1.03 & $4.50^{b}$ & 0.285 & 1.15 & 6.36 & 0.05 & 1.8 & 2.05 & 72 \\
328.15 & 0.348 & 1.07 & 4.39 & 0.308 & 1.18 & 6.30 & 0.05 & 1.8 & 1.98 & 54 \\
338.15 & 0.359 & 1.10 & 4.30 & 0.335 & 1.21 & 6.22 & 0.05 & 1.8 & 2.26 & 99 \\
\hline
\end{tabular}

${ }^{a}$ Units: $\tau_{\alpha}$ in ps, $\nu_{0, j}$ in $\mathrm{THz}, \gamma_{j}$ in $\mathrm{THz} ;{ }^{b}$ Parameter fixed; Italic values were interpolated; 


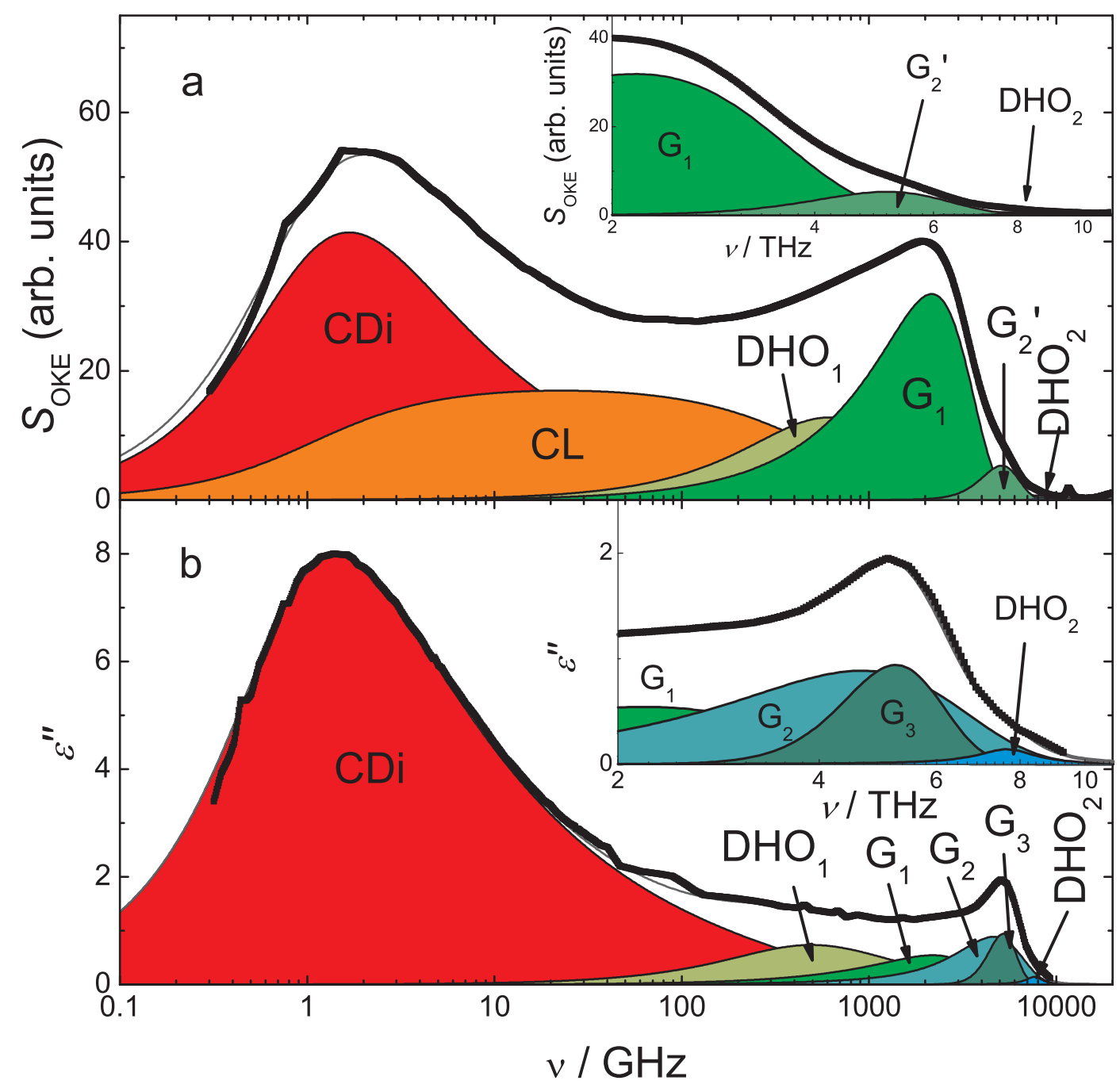

Figure S1: The imaginary part of (a) OKE and (b) DR spectra of $\mathrm{d}_{3}$-EAN at $25^{\circ} \mathrm{C}$. Black squares are resampled experimental data and gray lines represent the overall fit. Shaded areas indicate the contributions of the individual processes assuming a $\left(\mathrm{CDi}+\mathrm{CL}+\mathrm{DHO}_{1}\right.$ $\left.+\mathrm{G}_{1}+\mathrm{G}_{2}^{\prime}+\mathrm{DHO}_{2}\right)$ model for the OKE spectrum and a $\left(\mathrm{CDi}+\mathrm{DHO}_{1}+\mathrm{G}_{1}+\mathrm{G}_{2}+\mathrm{G}_{3}\right.$ $+\mathrm{DHO}_{2}$ ) model for the DR spectrum. Insets: expanded views of high frequency data. 

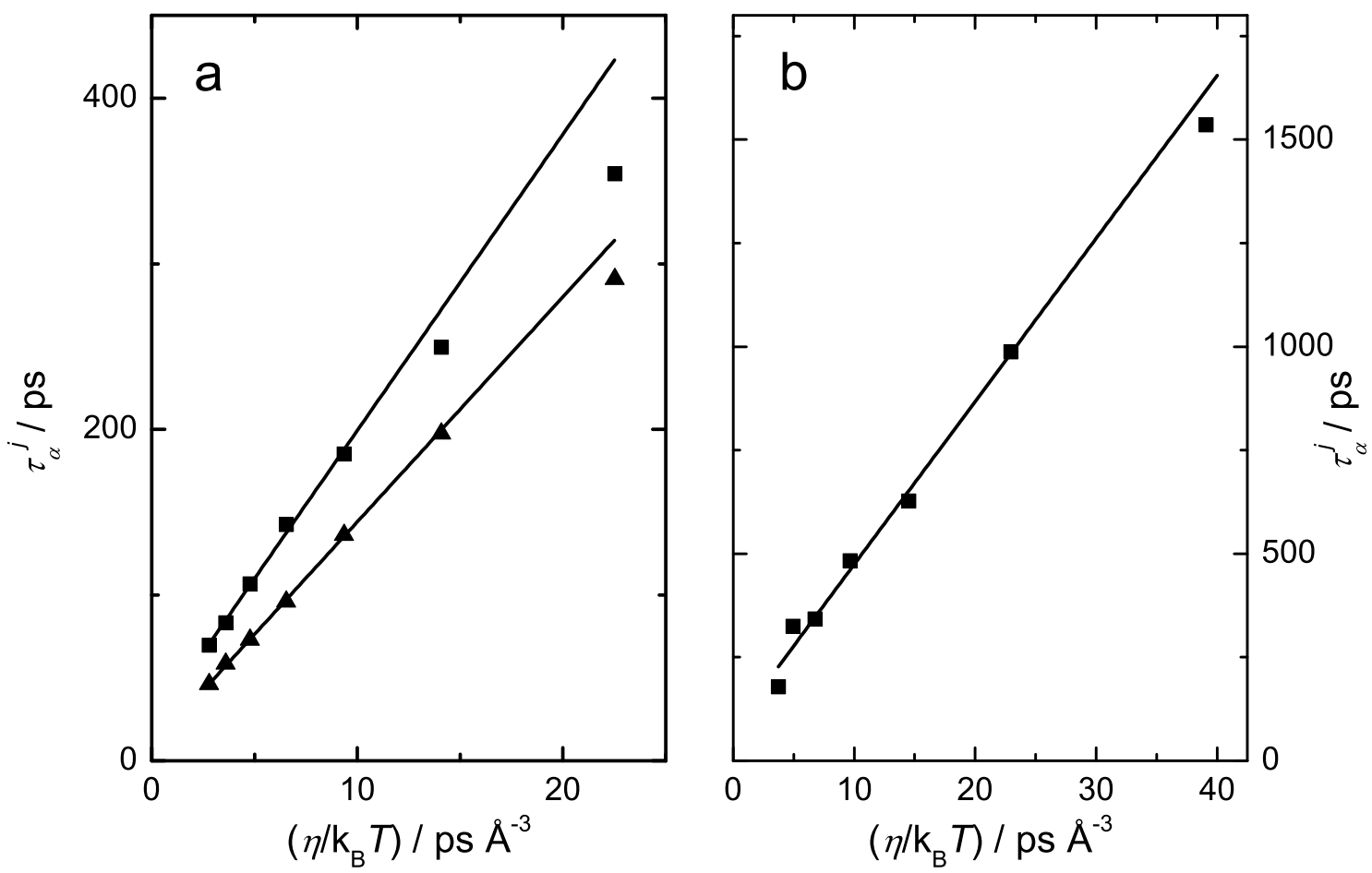

Figure S2: DR $(\boldsymbol{\square})$ and OKE $(\boldsymbol{\Delta})$ relaxation times of the $\alpha$-relaxation process, $\tau_{\alpha}^{j}$, of $(\mathbf{a})$ EAN (adapted from Fig. 6b of ref. 5) and (b) PAN as a function of $\eta / \mathrm{k}_{\mathrm{B}} T$.

\section{References}

(1) Weingärtner, H.; Knocks, A.; Schrader, W.; Kaatze, U. Dielectric Spectroscopy of the Room Temperature Molten Salt Ethylammonium Nitrate. J. Phys. Chem. A 2001, 105, 8646-8650.

(2) Oleinikova, A.; Bonetti, M. Critical Behavior of the Electrical Conductivitiy of Concentrated Electrolytes: Ethylammonium Nitrate in n-Octanol Binary Mixtures. J. Solution Chem. 2002, 31, 397-413.

(3) Smith, J. A.; Webber, G. B.; Warr, G. G.; Atkin, R. Rheology of Protic Ionic Liquids and Their Mixtures. J. Phys. Chem. B 2013, 117, 13930-13935.

(4) Bouzón Capelo, S.; Méndez-Morales, T.; Carrete, J.; López Lago, E.; Vila, J.; Cabeza, O.; Rodriguez, J. R.; Turmine, M.; Varela, L. M. Effect of Temperature and 
Cationic Chain Length on the Physical Properties of Ammonium Nitrate-Based Protic Ionic Liquids. J. Phys. Chem. B 2012, 116, 11302-11312.

(5) Turton, D. A.; Sonnleitner, T.; Ornter, A.; Walther, M.; Hefter, G.; Seddon, K. R.; Stana, S.; Plechkova, N.; Buchner, R.; Wynne, K. Structure and Dynamics in Protic Ionic Liquids: A Combined Optical Kerr-effect and Dielectric Relaxation Spectroscopy Study. Faraday Discuss. 2012, 154, 145-153. 